\title{
Spectral analysis of Swift long GRBs with known redshift
}

\author{
J. I. Cabrera ${ }^{1 \star}$, C. Firmani ${ }^{1,2}$, V. Avila-Reese ${ }^{1}$, G. Ghirlanda $^{2}$, G. Ghisellini ${ }^{2}$ and \\ L. Nava ${ }^{2,3}$ \\ ${ }^{1}$ Instituto de Astronomía, Universidad Nacional Autónoma de México, A.P. 70-264, 04510, México, D.F. \\ ${ }^{2}$ Osservatorio Astronomico di Brera, via E.Bianchi 46, I-23807 Merate, Italy \\ ${ }^{3}$ Universitá degli studi dell'Insubria, Dipartimento di Fisica e Matematica, via Valleggio 11, I-22100 Como, Italy
}

6 August 2021

\begin{abstract}
We study the spectral and energetics properties of 47 long-duration gamma-ray bursts (GRBs) with known redshift, all of them detected by the Swift satellite. Due to the narrow energy range $(15-150 \mathrm{keV})$ of the Swift-BAT detector, the spectral fitting is reliable only for fitting models with 2 or 3 parameters. As high uncertainty and correlation among the errors is expected, a careful analysis of the errors is necessary. We fit both the power law (PL, 2 parameters) and cut-off power law (CPL, 3 parameters) models to the time-integrated spectra of the 47 bursts, and we present the corresponding parameters, their uncertainties, and the correlations among the uncertainties. The CPL model is reliable only for 29 bursts for which we estimate the $\nu f_{\nu}$ peak energy $E_{\mathrm{pk}}$. For these GRBs, we calculate the energy fluence and the rest-frame isotropic-equivalent radiated energy, $E_{\gamma, \text { iso }}$, as well as the propagated uncertainties and correlations among them. We explore the distribution of our homogeneous sample of GRBs on the rest-frame diagram $E_{\mathrm{pk}}^{\prime}$ vs $E_{\gamma, \text { iso }}$. We confirm a significant correlation between these two quantities (the "Amati" relation) and we verify that, within the uncertainty limits, no outliers are present. We also fit the spectra to a Band model with the high energy power law index frozen to -2.3 , obtaining a rather good agreement with the "Amati" relation of non-Swift GRBs.
\end{abstract}

Key words: gamma rays: bursts — gamma rays: sources

\section{INTRODUCTION}

The Swift Mission (Gehrels et al. 2004) was designed mainly to rapidly detect, locate, and observe gamma-ray bursts (GRBs). After more than two years of operation, the Swift satellite has observed approximately $180 \mathrm{GRBs}$, for which more than 50 events have known redshifts reported in the Mission homepag£ 1 . In this paper we present results from an homogeneous spectral analysis that we have carried out to the Swift long-duration GRBs with known $z$ with the main aim of estimating the time-averaged spectral parameters of the prompt emission of these bursts, as well as related global quantities like the prompt energy fluence, $S_{\gamma}$, and the rest-frame isotropic-equivalent radiated energy, $E_{\gamma, \text { iso }}$.

Unfortunately, the spectral coverage of the Swift $\gamma$-ray detector, the Burst Alert Telescope (BAT, Barthelmv et al. $2005)$, is very narrow. The recommendation is to limit the spectral analysis to channels between $15 \mathrm{keV}$ and $195 \mathrm{keV}$, though the Swift-BAT's team suggests a more conservative

\footnotetext{
* E-mail: jcabrera@astroscu.unam.mx

1 http://swift.gsfc.nasa.gov/
}

upper limit of $150 \mathrm{keV}$ because, above this energy, the calibration is not yet sufficiently reliable. Thus, the BAT energy range is not broad enough to allow the spectra to be fitted unambiguously by the usual GRB broken power-law function of 4 parameters. Therefore, the inference of $\gamma$-ray spectral parameters and global quantities related to these parameters for the Swift GRBs is not an easy task.

A main concern of this task is the determination of the uncertainties of the spectral parameters and their propagation into the composite quantities. Due to the narrow energy range of Swift BAT we know a priori that the errors in the fitted spectral parameters will be large and correlated among them. Therefore, the appropriate handling of errors is crucial in order to make useful the spectral data generated by BAT. The effort is justified by the great value that an homogeneous sample has, where the spectral information for all the events is obtained with the same detector and analyzed with the same techniques and methods. This is the case of the growing-in-number sample of Swift GRBs.

The characterization of the time-averaged photon spectra of GRBs with known redshift is important for several reasons. On one hand, this characterization offers 
clues for understanding the radiation and particle acceleration mechanisms at work during the prompt phase of the bursts (see for recent reviews e.g., Zhang \& Mészáros 2004; Piran 2005; Mészáros 2006; Zhang 2007). On the other hand, the spectral parameters and the global quantities inferred from them $\left(S_{\gamma}, E_{\gamma, \text { iso }}\right.$, etc. $)$ are among the main properties that characterize GRBs. The study of the rest-frame correlations among these and other properties (e.g,. Amati et al. 2002; Atteia 2003; Ghirlanda, Ghisellini \& Lazzati 2004a; Yonetoku et al. 2004; Liang \& Zhang 2005; Firmani et al. 2006a) is currently allowing to learn key aspects of the nature of GRBs (e.g., Thompson 2006; Thompson, Rees \& Mészáros 2007; Ramirez-Ruiz \& Granot 2006). Furthermore, those correlations that are tight enough can be used to standardize the energetics of GRBs, making possible to apply GRBs for constructing the Hubble diagram up to unprecedentedly high redshifts (Ghirlanda et al. 2004b, 2006; Dai et al. 2004; Firmani et al. 2005, 2006b, 2007; Liang \& Zhang 2005; Xu, Dai \& Liang 2005; Schaefer 2007). These tight correlations, for a given cosmological model, can be used also to estimate the (pseudo)redshifts of GRBs with not measured redshifts, mainly from the extensive CGRO-BATSE database 2 (e.g., Llovd-Ronning. Frver \& Ramirez-Ruiz 2002; Atteia 2003). Thus, by combining the distribution in redshift of a large sample of GRBs with the observed flux distribution, inferences on the luminosity function and formation rate of GRBs can be obtained (Firmani et al. 2004; Guetta et al. 2005).

The outline of this paper is as follows. In $\S 2$ we explain the main steps of the spectral analysis carried out by us: selection of the sample ( $\S 2.1)$, spectral deconvolution (fit) procedure and fitting models ( $\S 2.2)$, and error analysis $(\S \S 2.3)$. The results of the spectral analysis are given in $\S 3$, along with the estimates of $S_{\gamma}$ and $E_{\gamma, \text { iso }}$ as well as some correlations among the obtained quantities. The summary and conclusions of the paper are presented in $\S 4$. In the Appendix the deconvolved spectra of the 47 GRBs studied here are plotted.

We adopt the concordance $\Lambda$ CDM cosmology with $\Omega_{M}=0.3, \Omega_{\Lambda}=0.7$, and $H_{0}=70 \mathrm{~km} \mathrm{~s}^{-1} \mathrm{Mpc}^{-1}$.

\section{SPECTRAL ANALYSIS}

The first step in our spectral analysis is the selection of the Swift sub-sample of GRBs to be studied ( $\$ \S 2.1)$. Then we proceed to the spectral deconvolution with XSPEC and determination of the GRB spectral parameters for two- and three-parameter photon models $(\S \S 2.2)$. By using these parameters, the energy fluence and isotropic-equivalent energy are calculated. Finally, we construct the error confidence ellipses for the spectral parameters and $E_{\gamma \text {,iso }}(\S \S 2.3)$.

The BAT is a large aperture $\gamma$-ray telescope. It consists of a $(2.4 \times 1.2) \mathrm{m}$ coded aperture mask supported one meter

2 In a recent paper, Li (2007a) showed that due both to a redshift degeneracy in the so-called Amati correlation Amati et al. 2002) and to its high scatter, is not possible to infer reliable (pseudo)redshifts from this correlation. above the $5200 \mathrm{~cm}^{2}$ area detector plane. The detector contains 32,768 individual cadmium-zinc-telluride detector elements, each one of $(4 \times 4 \times 2) \mathrm{mm}$ size. The effective efficiency of BAT starts at approximately $15 \mathrm{keV}$, attains a broad maximum at approximately $30-100 \mathrm{keV}$ and then falls off, attaining at $195 \mathrm{keV}$ the same level as at $15 \mathrm{keV}$. Due to the $1 \mathrm{~mm}$ thickness of the lead tiles, the BAT coded mask starts to be transparent around $150 \mathrm{keV}$. We reduce the BAT's data using the analysis techniques provided by the BAT's instrument tean 3 .

\subsection{The sample}

We analyse the time-integrated spectra of all available Swift GRBs with known redshift $z$. The list of events was taken from the daily updated compilation of J. Greiner for the period January 2005 to January 2007. The corresponding file spectra were loaded from the legacy NASA databast5. The total number of events in this list was 55. We exclude from the list the short-duration bursts $\left(T_{90}<2\right.$ s; GRB050509, GRB050724, and GRB061217) as well as those events with incomplete observational information (GRB050730, GRB060124, GRB060218, GRB060505 and GRB060512), leaving us with a preliminary list of 47 long GRBs with measured $z$.

The list of 47 events is given in Table 1 The first four columns of Table 1 give respectively the burst name, the class (see below), the redshift $z$, and the time $T_{\text {int }}$ that we use to integrate the spectrum. In principle $\mathrm{T}_{90}$ could be used as an integration time. Nevertheless two problems arise in this case. The first one has to do with the systematic underestimate of the fluence, hence of $E_{\gamma, \text { iso }}$. The second problem may be more important; the elimination of the (early and late) wings in the light curve can influence the estimates of the spectral parameters. For example the elimination of the late wing may harden artificially the spectrum. For these reasons we have fixed the integration time by a visual inspection of the light curve as the period where the signal is clearly identifiable in the background noise. A modest overestimate of $T_{\text {int }}$ does not influence on the values of the integrated parameters, though increases the noise. We would like to remark that $T_{\text {int }}$ is not a good estimate of the burst duration because its uncertainty is related to the identification of faint (some time extended) wings.

\subsection{Spectral fits}

It is well known that the GRB photon spectra are in general well described, in the energy range of $\sim 10 \mathrm{keV}$ to a few $\mathrm{MeV}$, by the so-called Band function (Band et al. 1993), which is a two smoothly connected power laws:

$$
\begin{array}{r}
N(E)=N_{0}\left(\frac{E}{100 \mathrm{keV}}\right)^{\alpha} \exp \left(-\frac{E}{E_{0}}\right), \\
E \leqslant E_{0}(\alpha-\beta) \\
=N_{0}\left[\frac{E_{0}(\alpha-\beta)}{100 \mathrm{keV}}\right]^{(\alpha-\beta)} \exp (\beta-\alpha)\left(\frac{E}{100 \mathrm{keV}}\right)^{\beta},
\end{array}
$$

\footnotetext{
3 http://heasarc.gsfc.nasa.gov/docs/swift/analysis/threads

4 http://www.mpe.mpg.de/ jcg/grbgen.html

5 ftp://legacy.gsfc.nasa.gov/swift/data/obs
} 


\begin{tabular}{|c|c|c|c|c|c|c|c|c|}
\hline GRB & $\begin{array}{c}\text { Class } \\
(2)\end{array}$ & $(3)$ & $\begin{array}{l}\mathrm{T}_{\mathrm{int}} \\
\mathrm{sec} \\
(4)\end{array}$ & $\begin{array}{c}\operatorname{Lg} N_{0}[\mathrm{sd}] \\
50 \mathrm{keV} \\
\quad(5)\end{array}$ & $\alpha[\mathrm{sd}]$ & $\begin{array}{l}e_{1} \\
\quad(7)\end{array}$ & $\begin{array}{l}e_{2} \\
\quad(8)\end{array}$ & $\begin{array}{c}\chi_{\text {red }}^{2} \\
(9)\end{array}$ \\
\hline 050126 & $\mathrm{~A}$ & 1.29 & 40.0 & $-2.656[0.026]$ & $-1.317[0.101]$ & $0.026(-1.00,0.01)$ & $0.106(-0.01,-1.00)$ & 1.399 \\
\hline 050223 & A & 0.5915 & 30.0 & $-2.652[0.028]$ & $-1.837[0.106]$ & $0.025(-0.99,0.13)$ & $0.109(-0.13,-0.99)$ & 0.902 \\
\hline 050315 & $\mathrm{D}$ & 1.949 & 129.0 & $-2.567[0.013]$ & $-2.101[0.055]$ & $0.012(-0.99,0.11)$ & $0.056(-0.11,-0.99)$ & 0.889 \\
\hline 050318 & $\mathrm{~A}$ & 1.44 & 34.5 & $-2.375[0.014]$ & $-1.968[0.057]$ & $0.012(-0.99,0.13)$ & $0.060(-0.13,-0.99)$ & 0.936 \\
\hline 050319 & $\mathrm{D}$ & 3.24 & 190.0 & $-3.100[0.034]$ & $-2.045[0.118]$ & $0.026(-0.98,0.17)$ & $0.119(-0.17,-0.98)$ & 0.687 \\
\hline 050401 & $\mathrm{~B}$ & 2.9 & 43.0 & $-1.667[0.012]$ & $-1.472[0.046]$ & $0.011(-1.00,-0.04)$ & $0.047(0.04,-1.00)$ & 1.060 \\
\hline 050416 & $\mathrm{D}$ & 0.6535 & 5.0 & $-2.225[0.055]$ & $-3.119[0.155]$ & $0.026(-0.95,0.30)$ & $0.182(-0.30,-0.95)$ & 0.971 \\
\hline 050505 & $\mathrm{~A}$ & 4.27 & 70.0 & $-2.418[0.018]$ & $-1.415[0.071]$ & $0.018(-1.00,-0.03)$ & $0.073(0.03,-1.00)$ & 0.789 \\
\hline 050525 & $\mathrm{~A}$ & 0.606 & 15.0 & $-0.932[0.003]$ & $-1.769[0.015]$ & $0.003(-1.00,-0.09)$ & $0.015(0.09,-1.00)$ & 2.934 \\
\hline 050603 & $\mathrm{~B}$ & 2.821 & 16.0 & $-1.347[0.010]$ & $-1.159[0.043]$ & $0.010(-1.00,-0.05)$ & $0.043(0.05,-1.00)$ & 0.913 \\
\hline 050803 & $\mathrm{~A}$ & 0.422 & 165.0 & $-2.868[0.022]$ & $-1.526[0.085]$ & $0.021(-1.00,0.04)$ & $0.088(-0.04,-1.00)$ & 0.882 \\
\hline 050814 & $\mathrm{~A}$ & 5.3 & 95.0 & $-2.750[0.030]$ & $-1.798[0.108]$ & $0.027(-0.99,0.12)$ & $0.117(-0.12,-0.99)$ & 1.036 \\
\hline 050820 & $\mathrm{C}$ & 2.61 & 32.0 & $-2.461[0.016]$ & $-1.689[0.067]$ & $0.015(-1.00,0.09)$ & $0.067(-0.09,-1.00)$ & 0.988 \\
\hline 050824 & $\mathrm{D}$ & 0.83 & 38.0 & $-3.102[0.081]$ & $-2.743[0.219]$ & $0.036(-0.95,0.30)$ & $0.241(-0.30,-0.95)$ & 1.0157 \\
\hline 050904 & $\mathrm{C}$ & 6.29 & 207.0 & $-2.603[0.009]$ & $-1.205[0.041]$ & $0.009(-1.00,-0.04)$ & $0.041(0.04,-1.00)$ & 1.070 \\
\hline 050908 & $\mathrm{~A}$ & 3.344 & 24.0 & $-2.685[0.031]$ & $-1.947[0.107]$ & $0.025(-0.99,0.17)$ & $0.114(-0.17,-0.99)$ & 0.969 \\
\hline $050922 \mathrm{C}$ & $\mathrm{B}$ & 2.198 & 10.0 & $-1.761[0.009]$ & $-1.371[0.038]$ & $0.009(-1.00,-0.02)$ & $0.038(0.02,-1.00)$ & 0.900 \\
\hline 051016B & $\mathrm{D}$ & 0.9364 & 6.0 & $-2.509[0.048]$ & $-2.331[0.145]$ & $0.030(-0.97,0.24)$ & $0.158(-0.24,-0.97)$ & 1.344 \\
\hline 051109 & $\mathrm{~A}$ & 2.346 & 43.0 & $-2.052[0.033]$ & $-1.626[0.124]$ & $0.032(-1.00,0$. & $0.129(-0.05,-1$ & 0.819 \\
\hline 051111 & $\mathrm{~B}$ & & 45.0 & $-2.080[0.008]$ & $-1.305[0.034]$ & $0.008(-1.00,-0$. & $0.035(0.04,-1$ & 0.764 \\
\hline 051221 & $\mathrm{C}$ & 0.547 & 3.1 & $-1.422[0.008]$ & $-1.397[0.037]$ & $0.008(-1.00,-0$ & $0.037(0.03,-1$ & 0.996 \\
\hline 060115 & $\mathrm{~A}$ & 3.53 & 128.0 & $-2.851[0.018]$ & $-1.771[0.071]$ & $0.016(-0.99,0.11)$ & $0.073(-0.11,-0$ & 0.875 \\
\hline 060206 & $\mathrm{~A}$ & 4.048 & 14.0 & $-2.184[0.011]$ & $-1.693[0.047]$ & $0.011(-1.00,0.06)$ & $0.049(-0.06,-1$. & 1.061 \\
\hline 060210 & $\mathrm{~A}$ & 3.91 & 278.0 & $-2.579[0.015]$ & $-1.544[0.059]$ & $0.015(-1.00,-0.01)$ & $0.060(0.01,-1.00)$ & 0.928 \\
\hline 060223 & $\mathrm{~A}$ & 4.41 & 14.8 & $-2.320[0.020]$ & $-1.775[0.078]$ & $0.019(-0.99,0.11)$ & $0.082(-0.11,-0.99)$ & 1.009 \\
\hline 060418 & $\mathrm{C}$ & 1.489 & 80.0 & $-1.993[0.006]$ & $-1.634[0.030]$ & $0.006(-1.00,-0.01)$ & $0.030(0.01,-1.00)$ & 0.815 \\
\hline 060502A & $\mathrm{B}$ & 1.51 & 34.0 & $-2.157[0.011]$ & $-1.431[0.045]$ & $0.011(-1.00,-0.01)$ & $0.046(0.01,-1.00)$ & 1.031 \\
\hline 060510B & $\mathrm{A}$ & 4.9 & 289.0 & $-2.839[0.011]$ & $-1.778[0.048]$ & $0.010(-1.00,0.07)$ & $0.049(-0.07,-1.00)$ & 0.643 \\
\hline 060522 & $\mathrm{~A}$ & 5.11 & 80.0 & $-2.829[0.024]$ & $-1.578[0.093]$ & $0.023(-1.00,0.08)$ & $0.098(-0.08,-1.00)$ & 1.188 \\
\hline 060526 & $\mathrm{C}$ & & & $-3.387[0.035]$ & $-1.924[0.140]$ & $0.028(-0.99,0.17)$ & $0.132(-0.17,-0.99)$ & 0.967 \\
\hline 060604 & $\mathrm{D}$ & & & & $-2.040[0$ & $0.060(-0.98$ & $0.290(-0.2$ & 0.804 \\
\hline 060605 & A & 3.711 & 37.0 & $-2.810[0.026]$ & $-1.474[0.105]$ & $0.025(-1.00,0.07)$ & $0.109(-0.07,-1.00)$ & 0.912 \\
\hline 060607 & $\mathrm{~A}$ & 3.082 & 127.0 & $-2.686[0.011]$ & $-1.436[0.046]$ & $0.010(1.00,0.02)$ & $0.047(-0.02,-1.00)$ & 0.947 \\
\hline 060614 & $\mathrm{C}$ & 0.125 & 154.0 & $-1.877[0.005]$ & $-1.936[0.024]$ & $0.004(-1.00,0.01)$ & $0.024(0.01,-1.00)$ & 1.072 \\
\hline 060707 & A & 3.425 & 81.0 & $-2.652[0.022]$ & $-1.667[0.082]$ & $0.021(-1.00,0.06)$ & $0.087(-0.06,-1.00)$ & 1.116 \\
\hline 060714 & $\mathrm{~A}$ & 2.71 & 135.0 & $-2.614[0.016]$ & $-1.935[0.063]$ & $0.013(-0.99,0.12)$ & $0.064(-0.12,0.99)$ & 1.161 \\
\hline 060729 & $\mathrm{C}$ & 0.54 & 134.0 & $-2.658[0.021]$ & $-1.870[0.083]$ & $0.019(-0.99,0.12)$ & $0.084(-0.12,-0.99)$ & 0.863 \\
\hline 060904B & $\mathrm{A}$ & 0.703 & 187.0 & $-3.059[0.022]$ & $-1.628[0.090]$ & $0.021(-1.00,0.10)$ & $0.090(-0.10,-1.00)$ & 0.855 \\
\hline 060906 & $\mathrm{~A}$ & 3.686 & 63.0 & $-2.386[0.020]$ & $-2.006[0.075]$ & $0.017(-0.99,0.14)$ & $0.078(-0.14,-0.99)$ & 1.114 \\
\hline 060908 & $\mathrm{~A}$ & 2.43 & 30.0 & $-2.040[0.009]$ & $-1.339[0.039]$ & $0.009(-1.00,-0.01)$ & $0.040(0.01,-1.00)$ & 0.864 \\
\hline 060926 & $\mathrm{D}$ & 3.2 & & & & $0.026(-0.97,0.24)$ & $0.151(-0.24,-0.97)$ & 0.972 \\
\hline 060927 & $\mathrm{~A}$ & & & & & $0.012(-1.00,0.07)$ & $0.055(-0.07,-1.00)$ & 1.158 \\
\hline 061007 & $\mathrm{C}$ & 1.261 & & & & $0.003(-0.99,-0.13)$ & $0.016(0.13,-0.99)$ & 0.523 \\
\hline 061110B & $\mathrm{C}$ & 3.44 & 147.0 & $-3.055[0.021]$ & $-1.069[0.091]$ & $0.021(-1.00,-0.05)$ & $0.091(0.05,-1.00)$ & 1.175 \\
\hline 061121 & $\mathrm{C}$ & 1.314 & 125.0 & $-1.936[0.004]$ & $-1.410[0.018]$ & $0.004(-1.00,-0.08)$ & $0.018(0.08,-1.00)$ & 0.450 \\
\hline 061222B & $\mathrm{A}$ & 3.355 & 57.0 & $-2.367[0.023]$ & $-1.962[0.084]$ & $0.020(-0.99,0.14)$ & $0.089(-0.14,-0.99)$ & 1.280 \\
\hline 070110 & $\mathrm{C}$ & 2.352 & 103.0 & $-2.806[0.016]$ & $-1.581[0.702]$ & $0.016(-1.00,0.06)$ & $0.070(-0.06,-1.00)$ & 1.007 \\
\hline
\end{tabular}

Table 1. The sample of Swift long GRBs with known $z$ analysed here (47 events). The $z$ and duration ( $\left.\mathrm{T}_{\mathrm{int}}\right)$ of the burst as well as the results from the PL photon model fit are reported. Second column refers to the class group assigned to the burst according to our spectral analysis (see text). The best fit values of the parameters and their one-dimensional standard deviations (sd) are given in columns 5 and 6; $e_{1,2}$ and $n_{1,2}$ in columns 7 and 8 are the semi-axis lengths and principal axes of the error ellipse, respectively. The reduced $\chi^{2}$ is reported in the last column $(\mathrm{dof}=58)$.

$$
E>E_{0}(\alpha-\beta)
$$

where $\alpha$ and $\beta$ are the photon indices of the low and high energy power laws, respectively, and $E_{0}$ is the $e$-folding (break) energy related to the peak energy in the $\nu f_{\nu}$ [or $\left.E^{2} f(E)\right]$ spectrum by $E_{\mathrm{pk}}=E_{0} \times(2+\alpha)$. Note that $E_{\mathrm{pk}}$ is well defined for $\alpha \geqslant-2$ and $\beta<-2$. The normalization $N_{0}$ is in photons $\mathrm{s}^{-1} \mathrm{~cm}^{-2} \mathrm{keV}^{-1}$.
The spectral range of BAT is narrower than that one of BATSE and other previous detectors. In the most recent update of the BAT-team homepage it is written that due to varying threshold levels in individual detectors, channels below $15 \mathrm{keV}$ should not be used for spectral analysis. Like-

\footnotetext{
6 http://swift.gsfc.nasa.gov/docs/swift/analysis/bat-digest.html
} 
wise, channels above $150 \mathrm{keV}$ are unreliable due to a lack of calibration data at those energies. Then, we are limited to the narrow energy band of $15-150 \mathrm{keV}$ for the spectral analysis of Swift bursts. This implies that model fitting with the 4-parameter Band function will not be reliable for most of these burst. Therefore, we proceed to fit the observed photon spectra to both the power law (PL) and cutoff power law (CPL) models. The former has only two parameters, the normalization, $N_{0}$, and the photon index, $\alpha$, and is given by:

$N(E)=N_{0}\left(\frac{E}{50 \mathrm{keV}}\right)^{\alpha}$

The CPL model implies one more parameter, an energy $e$-folding spectral break, $E_{0}$, related to $E_{\mathrm{pk}}$ in the same way as in the Band model (see above). The CPL model is defined as:

$N(E)=N_{0}\left(\frac{E}{50 \mathrm{keV}}\right)^{\alpha} \exp \left[-\frac{E}{E_{0}}\right]$

It is easy to verify that the CPL model (named also the Compton $-\mathrm{COMP}-$ model) is the Band model with $\beta \rightarrow$ $-\infty$.

As we will see later, the complex nature of the errors due to the small energy range of the spectra makes it more convenient to use the logarithms of $N_{0}$ and $E_{0}$ (or $E_{\mathrm{pk}}$ ) rather than the linear quantities. This choice reduces the asymmetry of the errors and of the confidence levels (CL) contour shapes. Therefore, we have introduced into XSPEC new models corresponding to Eqs. (2) and (3) (and also Eq. (1); see $\S \S 3.3)$ to carry out the fits with $\log N_{0}$ and $\log E_{\mathrm{pk}}$.

The spectral analysis was carried out by using the heasoft6.1.2 public software 7 . The first step in our analysis is to define the background-subtracted light curve from the corresponding data file. The photon counts at each channel are taken in the time interval $T_{\text {int }}$ where the main light curve is clearly above the background noise. The spectral file was corrected by position and systematic errors. Then, the photon counts at each channel is convolved with a response matrix, build up for a given event with the calibration matrix, in order to obtain the time-integrated energy spectrum. As mentioned above, the BAT detector is well calibrated only in the 15-150 keV energy range. Therefore, we used the 60 BAT channels in this range, with the default binning. The spectral fit to the different photon models mentioned above was performed with the heasoft package XPSEC (version 11.3.1).

For the GRBs for which the (CPL) $E_{\mathrm{pk}}$ may be estimated, we calculate the fluence, $S_{\gamma}$, corresponding to the observed spectral band (15-150 keV) only with the aim of completeness. In addition the rest isotropic-equivalent energy, $E_{\gamma, \text { iso }}$, is calculated from the observer bolometric fluence $S_{\gamma_{B}}$ corresponding to the spectral range $1-10000 \mathrm{keV}$ at rest:

$E_{\gamma, \text { iso }}=\frac{4 \pi S_{\gamma_{B}} d_{L}(z)^{2}}{(1+z)}$

where $d_{L}(z)$ is the cosmology-dependent luminosity distance.

7 http://heasarc.gsfc.nasa.gov/docs/software/lheasoft/download.html

8 We use the January 2007 matrix calibration provided by the Swift-BAT team.

\subsection{Errors from the fit and error propagation}

A main concern of the spectral deconvolution of BAT spectra is the expected large uncertainty and correlations among the fitted spectral parameters. Consequently, a careful interpretation of the errors is crucial to make usable and reliable the spectral analysis of Swift GRBs.

First, as mentioned above, we have carried out the fits using $\log N_{0}$ (PL model), and $\log N_{0}$ and $\log E_{\mathrm{pk}}(\mathrm{CPL}$ model), instead of the corresponding linear values. The errors in the linear parameters are very asymmetric and correlated among them in a very complex way; the corresponding regions at a given CL, as plotted with the XSPEC command steppar in the plane of two parameters, have shapes strongly deviating from ellipses. Instead, the errors and the CL regions when the fits are carried out with logarithmic quantities, tend to be more symmetric and ellipse-shaped, respectively. This facilitates the handling of the errors to calculate composite quantities and correlations among the GRB parameters.

The fit command of XSPEC, generates ellipsoidal confidence intervals that approximate the complex regions of joint variation of the parameters. These ellipsoids are characterized by the semi-axis lengths, $e_{i}$ (they are the square root of the variances), where $i$ is for each axis, and the corresponding principal axes (unitary vectors $n_{i}$ ). We use these data to calculate the covariance matrix, $C_{i j}$, and the elliptical boundary of the desired CL region in the subspace of interest (see e.g., Press et al. 1999). The diagonal elements of the matrix, $C_{i i}$, are the variances of each model parameter (two for the PL model and three for the CPL model), while the off-diagonal elements (covariances) show the correlations among the parameters.

The error propagation from the spectral quantities $\left(\log N_{0}, \alpha, \log E_{\mathrm{pk}}\right)$ to the composite quantities $\log E_{\mathrm{pk}}^{\prime}$ (being $E_{\mathrm{pk}}^{\prime}$ the peak energy at rest $\left.E_{\mathrm{pk}}^{\prime}=E_{\mathrm{pk}} \times[1+\mathrm{z})\right]$ ) and $\log E_{\gamma, \text { iso }}$ is performed in two steps. First, we have extracted sets of spectral quantities by a Monte Carlo method according to their elliptical CL. Then, for each set we have calculated the corresponding composite quantities as well as their covariance matrix averaging on the overall extractions. From this last covariance matrix the elliptical CL of the composite quantities is obtained.

\section{RESULTS}

In Table 1 we present the results from the spectral fit to the PL model for the whole sample of 47 long GRBs. Columns 1 to 4 give the burst name, the class group of the burst, $z$, and $\mathrm{T}_{\mathrm{int}}$. Columns 5 and 6 give the best fit values of the normalization, $\log N_{0}$, and the photon index, $\alpha$, with the corresponding one-dimensional symmetric standard deviations in brackets, as obtained with the XSPEC error command. In columns 7 and 8 we report the square root of the variances, $e_{i}$, together with the unitary vectors $\mathbf{n}_{\mathbf{i}}$ (principal axes), which provide respectively the semi-axis lenghts and directions of each axis $(i=1,2)$ of the joint error ellipse. The reduced $\chi^{2}$ is reported in column 9 .

As it may be appreciated from Table 1, the best-fit $\alpha$ is smaller than -2 for 7 bursts out of the 47 (GRB 060906 is marginal and it will be recovered by the CPL fitting). Most 
Spectral analysis of Swift GRBs with known z

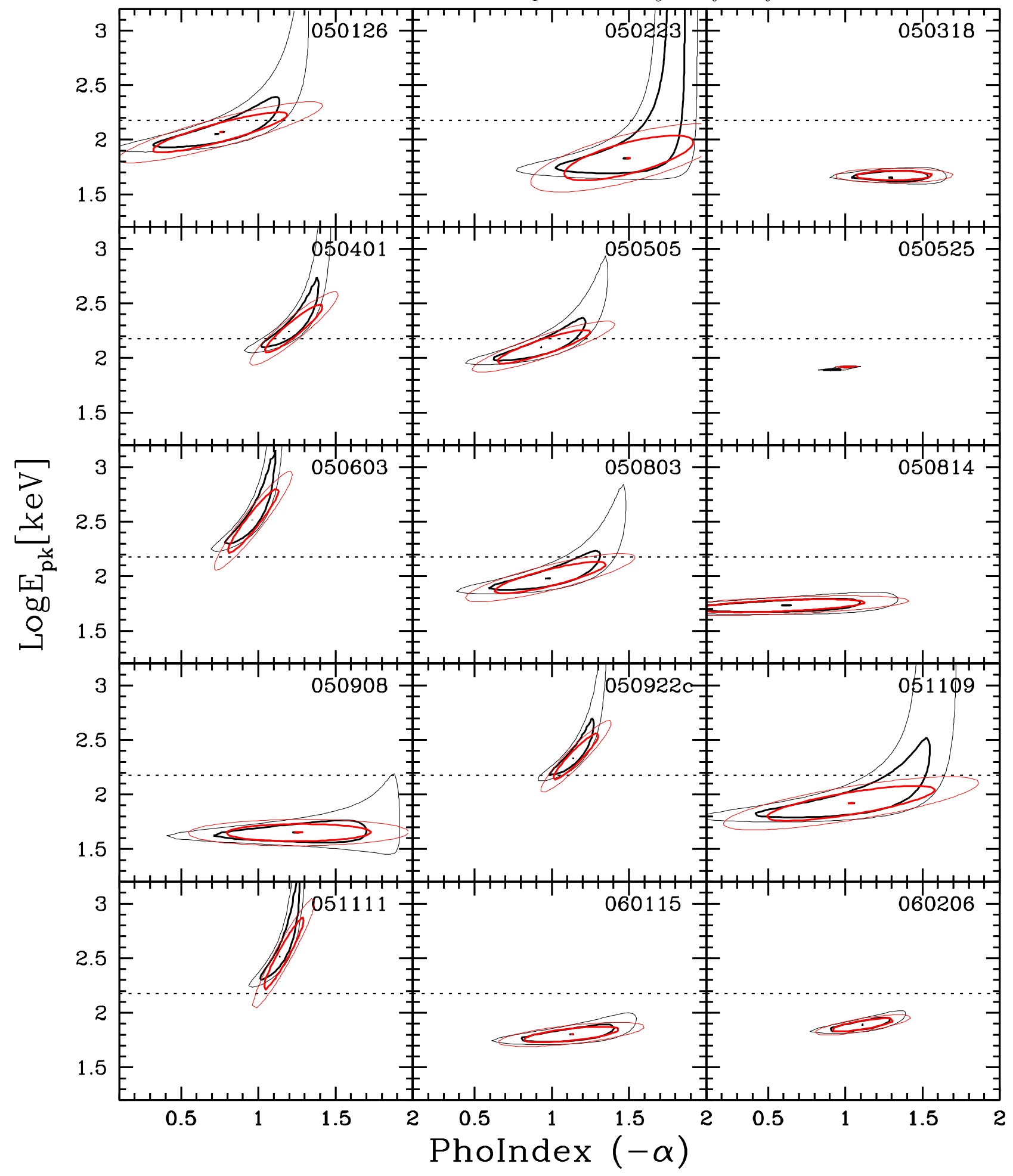

Figure 1. Error CL contours projected in the plane of the pair of variables $\log E_{\mathrm{pk}}-(-\alpha)$ for each one of the bursts in samples $A$ and B (CPL model). The irregular contours (black lines in the electronic version) were calculated with the XSPEC command steppar, while the elliptical contours (red lines) were constructed from the variances and principal axes given by the XSPEC command fit. Inner (thick line) and outer (thin line) contours in each case are for $\Delta \chi^{2}=1$ and 2.3 , respectively (see text). The dotted line indicates the level of $150 \mathrm{keV}$. 


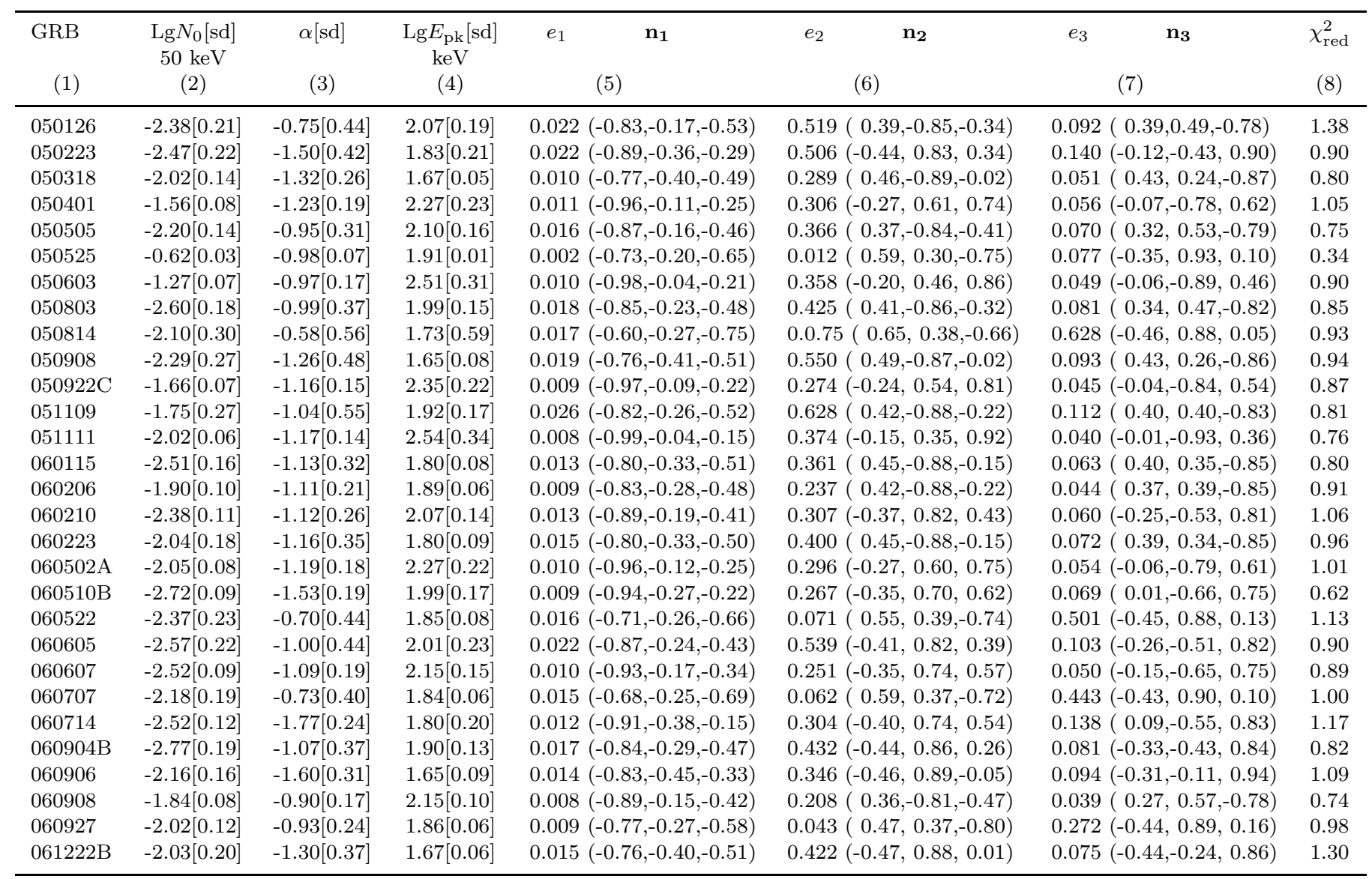

Table 2. Spectral fit results using the CPL photon model for the class A and B GRBs. The best fit values of the parameters and their one-dimensional standard deviations (sd) are given in columns $2-4 ; e_{1,2,3}$ and $n_{1,2,3}$ in columns $5-7$ are the semi-axis lengths and principal axes of the error ellipsoid, respectively. The reduced $\chi^{2}$ is reported in the last column (dof $=57$ ).

likely, the $\nu f_{\nu}$ spectra of these GRBs peak at energies below $15 \mathrm{keV}$. They could be related to the $\mathrm{X}$-ray rich events and X-ray Flashes discovered by BeppoSAX and HETE-2 (Kippen et al. 2001; Sakamoto et al. 2005).

We have also fitted the 47 GRB spectra to the CPL model and found that for 18 bursts the fit produces too large uncertainties and/or unphysical solutions. For these 18 cases the PL model is definitively more reliable than the CPL one. According to the results from our spectral fits (judged by the joint $68 \%$ CL contour errors), we classify the 47 Swift GRBs analysed here in four classes:

Class $A$ - The CPL-model fit is acceptable and $E_{\mathrm{pk}}$ is within the $15-150 \mathrm{keV}$ range.

Class $B$ - The CPL-model fit is acceptable, but $E_{\mathrm{pk}}>$ $150 \mathrm{keV}$.

Class $C$ - the CPL-model fit gives unreliable results, while the $\mathrm{PL}-$ model fit is acceptable and gives $\alpha>-2$, suggesting that $E_{\mathrm{pk}}$ is at energies $>150 \mathrm{keV}$.

Class $D$ - The CPL-model fit gives unreliable results, while the PL-model fit is acceptable and gives $\alpha<-2$, suggesting that $E_{\mathrm{pk}}$ is smaller than $15 \mathrm{keV}$.

The class of each GRB is indicated in the second column of Table 1. This classification can be easily understood after a visual inspection of the plots shown in Appendix and figures 1 and 2 In Figs. A1 A2 and A3 of the Appendix we present the deconvolved time-integrated $\nu f_{\nu} \quad\left[\right.$ or $\left.E^{2} f(E)\right]$ observed spectra of the 47 Swift GRBs analysed here. The CPL model was used for the spectral deconvolution. The error bars show the observations while the continuous lines show the fitted curves. The spectra of all the events that we classify in groups A and B show some curvature, evidence of a peak in the $E^{2} f(E)$ distribution. Nevertheless, in most of the cases the formal $F$-test analysis says that the spectral fits from the PL model to the CPL one (which introduces one more parameter, $E_{\mathrm{pk}}$ ) do not improve significantly. Therefore, one expects large uncertainties in the determination of $E_{\mathrm{pk}}$ for most of the events analyzed here. This is why it is important to carry out a carefully statistical analysis of the errors and to adequately propagate them for calculating composite quantities and inferring correlations.

Figures 1 and 2 show different contours of the CLs projected in the plane of the pair of variables $\log E_{\mathrm{pk}}$ vs. $(-\alpha)$ for each burst in groups A and B (CPL model). The irregular CL contours (black lines in the electronic version) were calculated with the XSPEC command steppar, while the elliptical CLs (red lines) were constructed from the joint variances and principal axes given by the XSPEC command $f i t$ (see $\S \S 2.3$; columns 5, 6, and 7 in Table 2 below). Thick lines show the CLs for $\Delta \chi^{2}=1$, while thin lines show the CLs for $\Delta \chi^{2}=2.3$. For $\Delta \chi^{2}=1$, the projections of the CL regions in each axis enclose $\approx 68 \%$ of the one-dimensional probability for the given parameter (one standard deviation), while 
Spectral analysis of Swift GRBs with known z

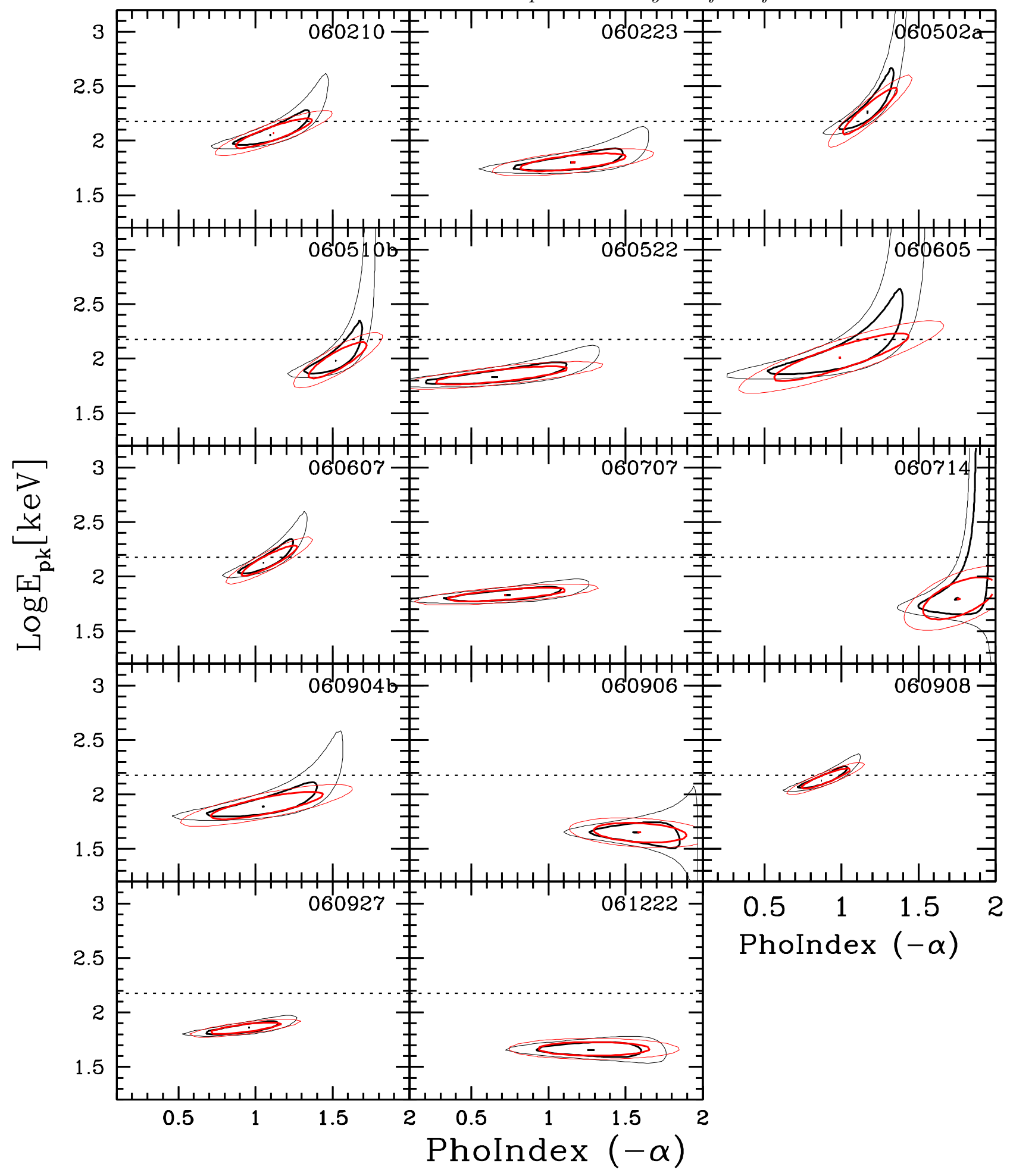

Figure 2. Continues Fig. 1

for $\Delta \chi^{2}=2.3$, the plotted region encompasses $\approx 68 \%$ of the probability for the joint variation of the two parameters (one standard deviation) (e.g., Press et al. 1999). As can be appreciated, the ellipses are a reasonable description of the CL regions in most cases, with the great advantage that they are handled analytically.

We see from Figs. 1 and 2 that for many of the GRBs, the $\log E_{\mathrm{pk}}$ and $\alpha$ spectral parameters are correlated (in- clined, thin ellipses). A similar situation arises for the other combination of parameters. Therefore, for this kind of data, a simple statistical quantification of the errors, such as the one-dimensional standard deviation, is not enough. This is why we have worked out the full covariance matrix calculated from the error ellipse. We use it to propagate errors in the calculation of $E_{\mathrm{pk}}^{\prime}$ and $E_{\gamma \text {,iso }}(\S \S 2.3)$. This is a crucial 


\begin{tabular}{|c|c|c|c|c|c|}
\hline $\begin{array}{l}\text { GRB } \\
\text { (1) }\end{array}$ & $\begin{array}{c}S_{\gamma}[\mathrm{s} . \mathrm{d}] \\
10^{-7} \text { erg cm }{ }^{2} \\
(2)\end{array}$ & $\begin{array}{c}\operatorname{Lg} E_{\text {iso }}^{\prime}[\mathrm{sd}] \\
10^{50} \mathrm{erg} \mathrm{s}^{-1} \\
(3)\end{array}$ & $\begin{array}{c}\operatorname{Lg} E_{\mathrm{pk}}^{\prime}[\mathrm{sd}] \\
\mathrm{keV} \\
(4)\end{array}$ & $\begin{array}{ll}e_{1} & \mathbf{n}_{1} \\
& \\
& (5)\end{array}$ & $\begin{array}{ll}e_{2} & \mathbf{n}_{2} \\
& (6)\end{array}$ \\
\hline 050126 & $8.55[1.82]$ & $1.70[0.18]$ & $2.42[0.18]$ & $0.062(0.73,-0.69)$ & $0.247(0.69,0.73)$ \\
\hline 050223 & $6.14[0.83]$ & $1.00[0.20]$ & $2.04[0.21]$ & $0.113(0.72,-0.69)$ & $0.266(0.69,0.72)$ \\
\hline 050318 & $12.90[0.54]$ & $2.07[0.11]$ & $2.06[0.04]$ & $0.107(1.00,0.03)$ & $0.044(-0.03,1.00)$ \\
\hline 050401 & $89.41[7.24]$ & $3.51[0.14]$ & $2.86[0.23]$ & $0.042(0.86,-0.51)$ & $0.261(0.51,0.86)$ \\
\hline 050505 & $25.79[3.06]$ & $3.13[0.12]$ & $2.82[0.16]$ & $0.040(0.79,-0.62)$ & $0.195(0.62,0.79)$ \\
\hline 050525 & $158.20[1.59]$ & $2.34[0.01]$ & $2.12[0.01]$ & $0.018(0.79,0.61)$ & $0.008(-0.61,0.79)$ \\
\hline 050603 & $74.60[8.18]$ & $3.55[0.22]$ & $3.10[0.30]$ & $0.061(0.81,-0.59)$ & $0.372(0.59,0.81)$ \\
\hline 050803 & $20.83[2.57]$ & $1.13[0.14]$ & $2.14[0.15]$ & $0.044(0.73,-0.68)$ & $0.199(0.68,0.73)$ \\
\hline 050814 & $14.60[1.16]$ & $2.94[0.11]$ & $2.53[0.06]$ & $0.114(0.93,0.36)$ & $0.047(-0.36,0.93)$ \\
\hline 050908 & $4.36[0.46]$ & $2.25[0.16]$ & $2.29[0.08]$ & $0.156(1.00,0.06)$ & $0.079(-0.06,1.00)$ \\
\hline $050922 \mathrm{C}$ & $16.97[0.94]$ & $2.63[0.14]$ & $2.86[0.22]$ & $0.034(0.85,-0.53)$ & $0.257(0.53,0.85)$ \\
\hline 051109 & $35.27[5.85]$ & $2.80[0.19]$ & $2.44[0.16]$ & $0.238(0.77,0.64)$ & $0.077(-0.64,0.77)$ \\
\hline 051111 & $37.32[3.59]$ & $2.80[0.22]$ & $2.96[0.34]$ & $0.056(0.84,-0.54)$ & $0.399(0.54,0.84)$ \\
\hline 060115 & $16.04[1.07]$ & $2.78[0.10]$ & $2.46[0.07]$ & $0.121(0.84,0.54)$ & $0.044(-0.54,0.84)$ \\
\hline 060206 & $8.29[0.35]$ & $2.59[0.06]$ & $2.59[0.06]$ & $0.086(0.72,0.70)$ & $0.024(-0.70,0.72)$ \\
\hline 060210 & $69.24[3.74]$ & $3.52[0.10]$ & $2.76[0.14]$ & $0.030(0.81,-0.59)$ & $0.170(0.59,0.81)$ \\
\hline 060223 & $6.54[0.52]$ & $2.56[0.12]$ & $2.53[0.08]$ & $0.139(0.86,0.50)$ & $0.052(-0.50,0.86)$ \\
\hline $060502 \mathrm{~A}$ & $22.87[1.66]$ & $2.42[0.14]$ & $2.67[0.22]$ & $0.039(0.85,-0.53)$ & $0.259(0.53,0.85)$ \\
\hline 060510B & $38.64[2.88]$ & $3.53[0.13]$ & $2.76[0.17]$ & $0.046(0.80,-0.59)$ & $0.207(0.59,0.80)$ \\
\hline 060522 & $10.49[1.04]$ & $2.78[0.09]$ & $2.63[0.08]$ & $0.120(0.74,0.67)$ & $0.036(-0.67,0.74)$ \\
\hline 060605 & $5.33[1.12]$ & $2.31[0.20]$ & $2.69[0.22]$ & $0.074(0.74,-0.67)$ & $0.289(0.67,0.74)$ \\
\hline 060607 & $24.91[1.58]$ & $2.94[0.10]$ & $2.76[0.15]$ & $0.026(0.83,-0.55)$ & $0.173(0.55,0.83)$ \\
\hline 060707 & $16.26[1.13]$ & $2.70[0.08]$ & $2.48[0.06]$ & $0.097(0.81,0.59)$ & $0.036(-0.59,0.81)$ \\
\hline 060714 & $30.58[3.96]$ & $3.11[0.15]$ & $2.37[0.20]$ & $0.111(0.85,-0.53)$ & $0.219(0.53,0.85)$ \\
\hline 060904B & $14.78[1.91]$ & $1.44[0.13]$ & $2.13[0.13]$ & $0.174(0.71,0.71)$ & $0.047(-0.71,0.71)$ \\
\hline 060906 & $23.82[1.92]$ & $3.16[0.14]$ & $2.32[0.09]$ & $0.142(0.98,-0.20)$ & $0.087(0.20,0.98)$ \\
\hline 060908 & $26.56[1.11]$ & $2.78[0.07]$ & $2.68[0.10]$ & $0.016(0.81,-0.59)$ & $0.123(0.59,0.81)$ \\
\hline 060927 & $11.66[0.46]$ & $2.92[0.06]$ & $2.68[0.06]$ & $0.077(0.72,0.70)$ & $0.022(-0.70,0.72)$ \\
\hline 061222B & $22.35[1.23]$ & $2.97[0.14]$ & $2.31[0.06]$ & $0.137(1.00,0.01)$ & $0.064(-0.01,1.00)$ \\
\hline
\end{tabular}

Table 3. Calculated fluence and rest-frame isotropic and peak energies for our GRB groups A and B. The corresponding one-dimensional standard deviations are given between square brackets. The last 2 columns give the estimated semi-axis lengths $\left(e_{1,2}\right)$ and principal

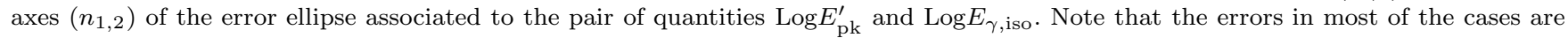
correlated significantly (see also the plotted ellipses in Figs. 4 and 5).

step when using the data, for instance, for establishing correlations among the spectral or composite quantities ( $\S 3.3)$.

The CPL-model fittings for GRB groups A and B are presented in Table 2 Column 1 gives the burst names. Columns 2, 3, and 4 report the best fit values of $\log N_{0}$, $\alpha$, and $\log E_{\mathrm{pk}}$ with their corresponding one-dimensional standard deviations, as calculated with the XSPEC error command. In columns 5,6 , and 7 we report the lengths $e_{i}$ of each semi-axis together with the unitary vectors $\mathbf{n}_{\mathbf{i}}$ (principal axes), which provide the directions of each axis $(i=1,2,3)$. The reduced $\chi^{2}$ is reported in column 8 (dof $=$ $57)$.

Finally, in Table 3 we report the calculated values for $S_{\gamma}, \log E_{\gamma, \text { iso }}$, and $\log E_{\mathrm{pk}}^{\prime}$, as well as the corresponding propagated errors for the samples $\mathrm{A}$ and $\mathrm{B}$, using the CPLmodel fit results (see $\S 2.3$ for the used error propagation procedure). Column 1 gives the burst name. Columns 2 and 3 give respectively the values of $S_{\gamma}$ (in the observed range $15-150 \mathrm{keV}$ ) and of $E_{\gamma \text {,iso }}$ (extrapolated to the rest-frame energy range $1-10000 \mathrm{keV}$ ) corresponding to the best fit spectral parameters. Column 4 gives the value of the restframe peak energy, $E_{\mathrm{pk}}^{\prime}$. The one-dimensional standard de-

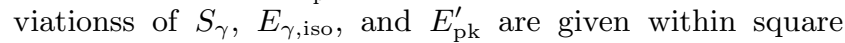
brackets. However, due to the high correlation among the errors the simple standard deviation is not enough to char- acterize statistically the errors. Thus, in columns 5 and 6 we report the error ellipse elements (semi-axis lenghts, $e_{i}$, and principal axes $\mathbf{n}_{\mathbf{i}}, i=1,2$ ) associated to the pair of

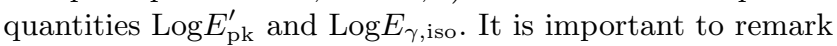
that the correlation between the uncertainties of $\log E_{\mathrm{pk}}^{\prime}$ and $\log E_{\gamma, \text { iso }}$ is not entirely due to the correlation among $\log N_{0}, \alpha$, and $\log E_{\mathrm{pk}}$. For instance an increase in $\log E_{\mathrm{pk}}$ alone is able to produce an increase in both $\log E_{\mathrm{pk}}^{\prime}$ and $\log E_{\gamma, \text { iso }}$. This effect will turn out to be helpful in the next section, where we will study how $\log E_{\mathrm{pk}}^{\prime}$ and $\log E_{\gamma \text {,iso }}$ do correlate in the entire GRB sample.

The median and dispersion (quartiles) of the bestfit CPL parameter $\alpha$ for the $24+5$ classes $\mathrm{A}+\mathrm{B}$ GRBs are $-1.12_{-0.14}^{+0.15}$, which is in agreement with the results from BATSE bright-GRB (Kaneko et al. 2006) and HETE-2 GRB (Barraud et al. 2003; Sakamoto et al. 2005) time-integrated spectra. Our $\alpha$ distribution is in particular similar to the one from HETE-2, with only $\sim 25 \%$ of the bursts having $\alpha \lesssim-1.2$. The median and quartiles of the $\log E_{\mathrm{pk}}[\mathrm{keV}]$ parameter are $1.91_{-0.11}^{+0.19}$. Unfortunately, due to the low energy limit of the Swift-BAT, the sample analysed here covers only the $\sim 15 \%$ fraction of bursts in the (CPL-model) $E_{\mathrm{pk}}$ distribution inferred for BATSE bright bursts (Kaneko et al. 2006).

The distribution of $E_{\mathrm{pk}}^{\prime}$ (at rest) is much broader than 
Spectral analysis of Swift GRBs with known z
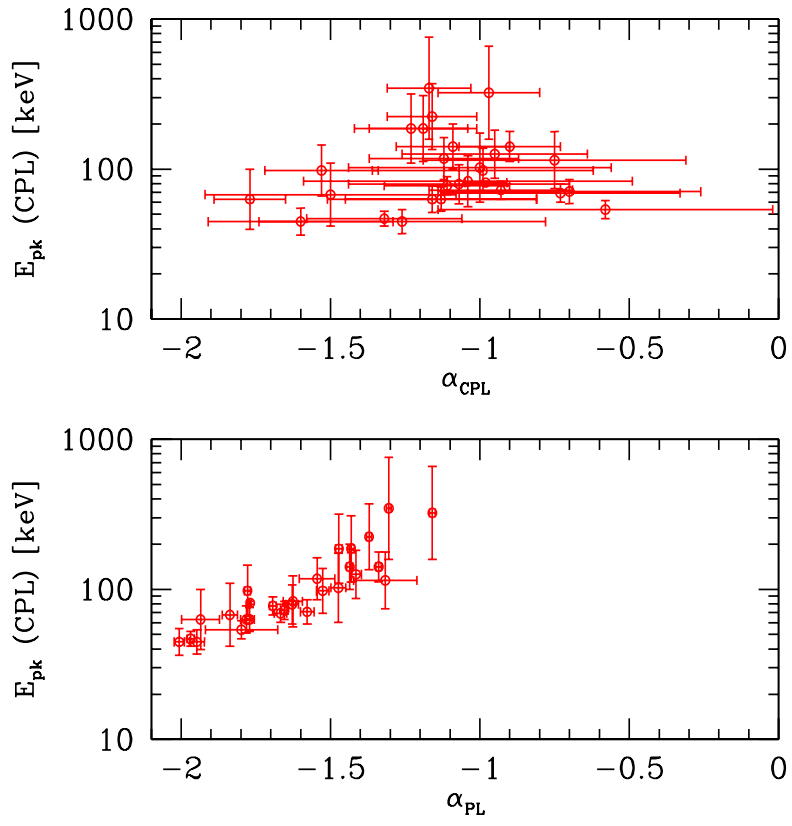

Figure 3. Top panel: the observed $E_{\mathrm{pk}}$ as derived by the CPL fit as a function of the spectral index $\alpha_{\mathrm{CPL}}$ of the same fit. Bottom panel: $E_{\mathrm{pk}}$ (as derived by the CPL fit) as a function of the spectral index $\alpha_{\mathrm{PL}}$ of the single power law fit. The correlation of $E_{\mathrm{pk}}$ with $\alpha_{\mathrm{PL}}$ is induced by $E_{\mathrm{pk}}$ entering in the Swift energy band (see text), and cannot be used to infer $E_{\mathrm{pk}}$ when knowing only $\alpha_{\mathrm{PL}}$.

the one of $E_{\mathrm{pk}}$ (the median and quartiles of $\log E_{\mathrm{pk}}^{\prime}[\mathrm{keV}]$ are $\left.2.54_{-0.21}^{+0.22}\right)$. This is expected due to the broad distribution in redshfits of the Swift bursts analysed here. For our $24+5$ classes A+B GRBs, the $z$ distribution has a broad maximum at $z \sim 3-4$; the median and quartiles are $3.08_{-1.5}^{+0.82}$. The median and quartiles of $\log E_{\gamma, \text { iso }}\left[10^{50} \mathrm{erg}\right]$ for our $24+5$ classes A+B GRBs are $2.78_{-0.44}^{+0.30}$. For the 8 long-duration Swift GRBs in common with the compilation presented in Amati (2006b), the values of $E_{\mathrm{pk}}^{\prime}$ reported here and in that paper are similar, but those of $E_{\gamma \text {,iso }}$ are systematically larger in Amati (2006b). The latter difference is probably due to the fact that Amati (2006b) did not use for most of those GRBs the spectral information from the Swift satellite but from other space missions (Konus, HETE-2), which allowed to find a fitting with the Band model; as will be discussed in $\S \S 3.3$, this implies typically a larger $E_{\gamma \text {,iso }}$ with respect to the case a CPL model is used.

\subsection{Bursts detected also by other instruments}

Among the bursts listed in Tables 2 and 3 there are four GRBs which have been detected by other instruments besides Swift. Three of them have been detected by KonusWind (GRB 050401, 050603 and 051109A) and one by HETE-2 (GRB 050922C).

For GRB 050401 the Konus-Wind results reported by Golenetskii et al. (2005a) concern the spectral parameters for the two peaks displayed by its light curve, fitted with a Band model in the energy range [20-2000] keV. The first peak had $\alpha=-1.15 \pm 0.16, \beta=-2.65 \pm 0.31$ and $E_{\mathrm{pk}}=$ $132 \pm 16 \mathrm{keV}$, while the second peak is described by $\alpha=$ $-0.83 \pm 0.21, \beta=-2.37 \pm 0.14$ and $E_{\mathrm{pk}}=119 \pm 26 \mathrm{keV}$.
Our analysis with the Swift data (see Tab. 3) for the time integrated spectrum and a CPL model yielded $\alpha=-1.23 \pm$ 0.19 and $E_{\mathrm{pk}}=186_{-76}^{+130} \mathrm{keV}$. Within the (relatively large) errors, the results are consistent.

The Konus-Wind data of GRB 050603 have been fitted by Golenetskii et al. (2005b) with a Band model in the [203000] keV energy range, with $\alpha=-0.79 \pm 0.06, \beta=-2.15 \pm$ 0.09 and $E_{\mathrm{pk}}=349 \pm 28 \mathrm{keV}$. Our analysis with a CPL model gives $\alpha=-1.23 \pm 0.19$ and $E_{\mathrm{pk}}=323_{-164}^{+340} \mathrm{keV}$. The derived low energy spectral index is somewhat softer, while the value of $E_{\mathrm{pk}}$ is consistent.

GRB $050922 \mathrm{C}$ was detected by HETE-2 with the FREGATE instruments in the 7-30, 7-80, and 30-400 keV bands. It was fitted with a CPL law model by Crew et al. (2005) with $\alpha=-0.83_{-0.26}^{+0.23}$ and $E_{\mathrm{pk}}=130.5_{-26.8}^{+50.9} \mathrm{keV}$, to be compared with our values of $\alpha=-1.16 \pm 0.15$ and $E_{\mathrm{pk}}=224_{-89}^{+148}$ $\mathrm{keV}$. Again, within the errors, there is consistency.

GRB 051109, detected by Konus-Wind and fitted with a CPL model by Golenetskii et al. (2005c) in the [20-500] $\mathrm{keV}$ energy range, showed $\alpha=-1.25_{-0.59}^{+0.44}$ and $E_{\mathrm{pk}}=$ $161_{-58}^{+224} \mathrm{keV}$. This should be compared with our $\alpha=-1.04 \pm$ 0.55 and $E_{\mathrm{pk}}=83_{-27}^{+40} \mathrm{keV}$. Even in this case there is consistency, within the relatively large errors.

\subsection{Peak energy vs spectral index}

In Fig. 3 we show $E_{\mathrm{pk}}$, derived with the CPL fit, as a function of the spectral index $\alpha_{\mathrm{CPL}}$ of the CPL fit (top panel) and as a function of $\alpha_{\mathrm{PL}}$, the index derived when fitting with a single power law. As can be seen, although there is no relation between $E_{\mathrm{pk}}$ and $\alpha_{\mathrm{CPL}}$, a correlation appears when fitting with a single power law. This correlation, however, has no physical meaning, since it is the result of the attempt of the single power law to account for the data point above the peak, with smaller flux (in $\nu f_{\nu}$ ). As $E_{\mathrm{pk}}$ decreases, a larger fraction of data lies above the peak, inducing the single power law to steepen. This also means that this effect vanishes when $E_{\mathrm{pk}}$ is outside the Swift energy range, therefore if we do not know $E_{\mathrm{pk}}$, we cannot use this correlation to infer it. Furthermore, note the difference in the derived values of the spectral indices. As expected for these bursts for which we could derive $E_{\mathrm{pk}}$, the index $\alpha_{\mathrm{PL}}$ is systematically softer than $\alpha_{\mathrm{CPL}}$. Given the obvious result of this subsection, we have considered unnecessary to show the upper panel of Fig. 3 with the $68 \%$ CL error ellipses. A complete treatment of the errors in this sense confirms the previous result.

\subsection{Correlation between $E_{\mathrm{pk}}^{\prime}$ and $E_{\gamma \text {,iso }}$}

In Fig. $4 E_{\mathrm{pk}}^{\prime}$ is plotted vs $E_{\gamma \text {,iso }}$ for the class A (red-line ellipses) and class B (green-line ellipses) Swift GRBs. The ellipses correspond to the joint $68 \%$ CL error regions calculated as explained in $\S \S 2.3$. The dotted straight lines encompass the $3 \sigma$ scatter of the updated Amati correlation presented in Ghirlanda (2007) for 49 GRBs; the central thin solid straight line is the best fit for that sample. A visual inspection shows that the Swift data analyzed in this paper are indeed correlated in the $E_{\mathrm{pk}}^{\prime}-E_{\gamma, \text { iso }}$ diagram. Taking into account the uncertainties, no GRB may be classified as outlier. Eight GRBs show their central point above the 


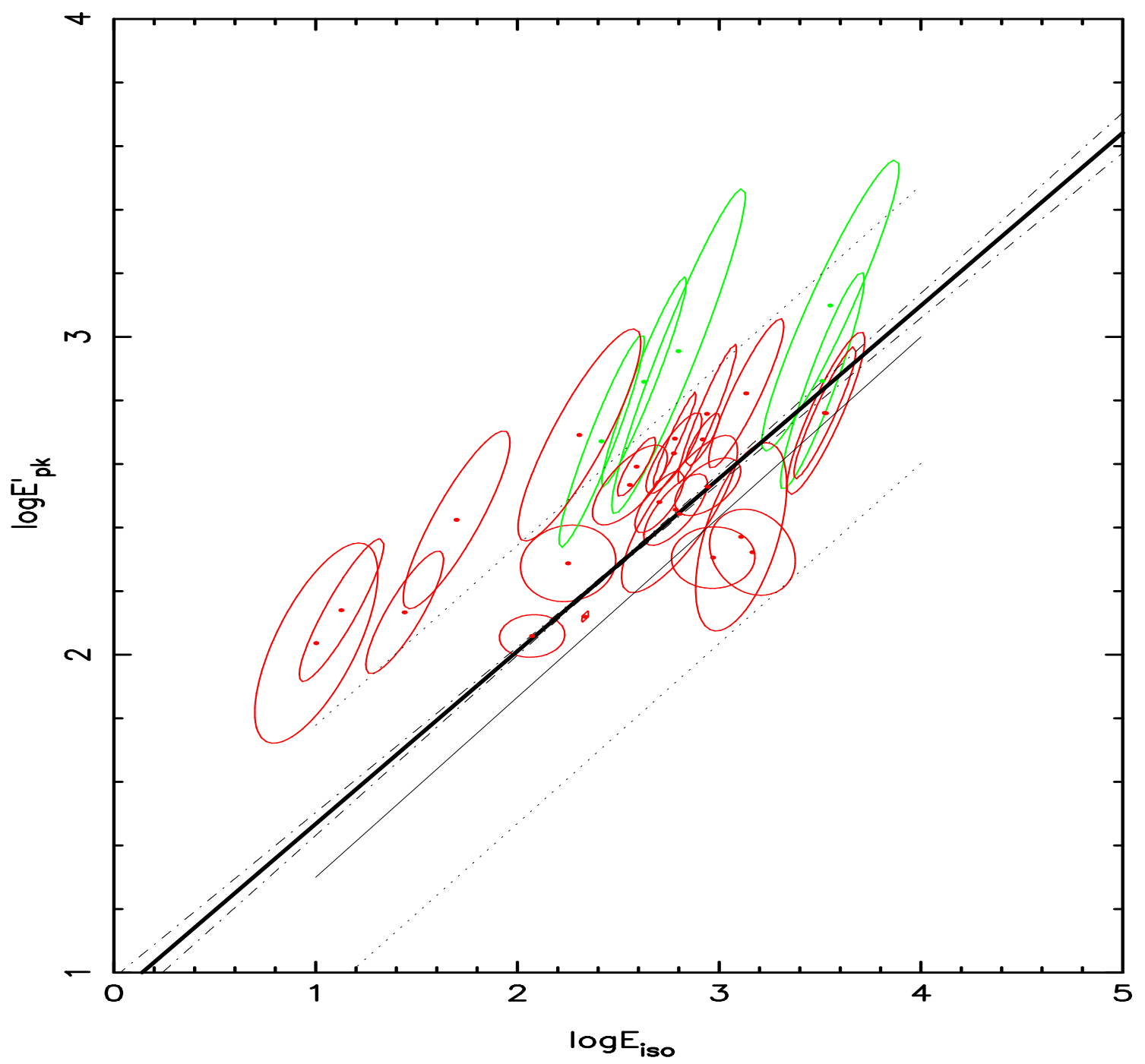

Figure 4. Correlation between $E_{\mathrm{pk}}^{\prime}$ and $E_{\gamma, \text { iso }}$ (rest frame) as obtained from spectral fits using the CPL model for the class A (red ellipses) and B (green ellipses) bursts. The ellipses correspond to the joint $68 \%$ CL error regions of each data point. The dotted lines encompass the $3 \sigma$ scatter of the updated Amati correlation presented in Ghirlanda (2007) for 49 GRBs; the thin solid line is the best fit for that sample. The thick solid line is the best fit that we find for the Swift data presented here taking into account the correlated errors, and the dot-dashed line is for the $1 \sigma$ uncertainty, computed in the barycentre of the data points.

$3 \sigma$ strip, but taking into account the $68 \%$ CL contours as well as the fact that the spectral fitting was performed using a CPL model instead of the Band one (see below), make such GRBs compatible with the $3 \sigma$ strip. Notice that the 5 (marginal) class B events are not outliers. Therefore, we may include them for a fit along with the 24 class A events.

The thick solid line in Fig. 4 is the best fit that we find for the 29 class A and B GRBs. The linear fit has been performed by an iterative minimum $\chi^{2}$ method, where the coordinate origin of the data is shifted to the barycentre, and the uncertainty component of each datapoint is calculated from the corresponding error ellipse in the orthogonal direction of the straight fitted line. The dot-dashed line curves describe the standard error of the mean values of the fit parameters computed in the barycentre of the data points. The best fit in logarithmic quantities is:

$\log \left(\frac{E_{\mathrm{pk}}^{\prime}}{\mathrm{keV}}\right)=(2.25 \pm 0.01)+(0.54 \pm 0.02) \log \left(\frac{E_{\gamma, \text { iso }}}{10^{52.44} \mathrm{erg}}\right)$
We point out the weight of GRB050525 in determining the best fit due to its very-reduced spectral parameter uncertainties. We have also carried out the linear fit for only the 24 class A GRBs:

$\log \left(\frac{E_{\mathrm{pk}}^{\prime}}{\mathrm{keV}}\right)=(2.23 \pm 0.01)+(0.54 \pm 0.02) \log \left(\frac{E_{\gamma, \text { iso }}}{10^{52.42} \mathrm{erg}}\right)$

As expected, the fit is very similar to the one that includes the 5 class B GRBs.

Thus, a correlation between $E_{\mathrm{pk}}^{\prime}$ and $E_{\gamma \text {,iso }}$ is confirmed for 29 long GRBs observed by the Swift satellite and homogeneously analyzed by us. The values of the power-law index $(\alpha \sim 0.54)$ and normalization $(K \sim 100 \mathrm{keV}$ for

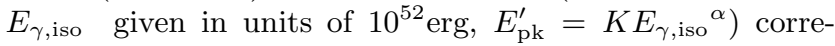
sponding to the best fit, lie in the ranges of values found with different previous samples (see for a review of previous studies in Amati 2006a). It is encouraging that with the Swift data, as analysed here, one obtains an $E_{\mathrm{pk}}^{\prime}-E_{\gamma \text {,iso }}$ 
correlation fully consistent with the one obtained for GRBs observed with other satellite detectors (the so-called Amati relation; Amati et al. 2002, see also Amati 2006a and Ghirlanda 2007 for the most recent updates), which had a much broader energy (spectral) range than BAT. The errors in the fit parameters reported in eqs. (5) and (6) are at the $68 \%$ CL. These errors are somewhat larger than those reported in Ghirlanda (2007) and Amati (2006a; note that therein the errors are at the $90 \% \mathrm{CL}$ ).

In general, the scatter around the correlation obtained here is within the range of different previous determinations of the Amati correlation. The value of the reduced $\chi^{2}$ for the fit with the 29 class $\mathrm{A}+\mathrm{B}$ GRBs is $\chi_{\nu}^{2}=379 / 27=14$ close to the values $\chi_{\nu}^{2}=493 / 41=12$ and $\chi_{\nu}^{2}=530 / 47=$ 11.3 obtained for larger samples (43 and 49 GRBs) by Ghirlanda et al. (2006) and Ghirlanda (2007), respectively. We have also estimated the variance of our fit, $s^{2}$ (see e.g., Bevington \& Robinson 1992, Chapter 11), by taking into account the joint variance of the data, i.e. the elliptical CL contours. For the fit using the $24+5 \mathrm{GRBs}$, we find $s=0.13$ $($ dof $=27)$. Note that for calculating the deviations, we use orthogonal distances to the best-fitting line rather than distances projected in the $\log E_{\mathrm{pk}}^{\prime}(\mathrm{Y})$ axis. An estimate of the vertical $1 \sigma$ spread of the data around the best-fitting model would be $\sigma_{\log E_{\mathrm{pk}}^{\prime}}=0.15$, in agreement with previous estimates (see e.g., Amati 2006a).

The virtue of the Swift sample analysed here is its homogeneity, in the sense of observations, reduction, and analysis. Previous samples used to infer the $E_{\mathrm{pk}}^{\prime}-E_{\gamma, \text { iso }}$ correlation were constructed from different detectors, with an information collected from different authors, who used different data processing and assumptions. This heterogeneity in the data should introduce a spurious scatter. In spite that the data used here have large observational errors, the dispersion of our fit lies within the range of fits reported previously. This is namely due to the homogeneity of the sample and because, having taken into account the whole error ellipses associated to the data points, it results that the major-axis orientation of most of these ellipses is close to the direction of the correlation line.

Examining in more detail Fig. 4, one sees that the normalization of the correlation found here [eq. (5)] is slightly higher (by $\approx 0.1$ dex at the sample median value of $E_{\gamma, \text { iso }}$ ) than the previously established one. This is indeed expected. Due to the narrow energy range of BAT, we had to force the GRB spectra to be fitted by the CPL model, while most spectra could have been better described by the Band model, if higher energies had been detected. It is known that when fitting the CPL model to a spectrum whose shape is actually described by the Band model, then (i) $E_{\mathrm{pk}}$ is larger than it would otherwise be, and (ii) the low-energy powerlaw index $\alpha$ is more negative than it would otherwise be (Band et al. 1993; Kaneko et al. 2006). On the other hand, (iii) in the CPL model the $\nu f_{\nu}$ spectrum is cut-off exponentially after the break energy $E_{0}$, while in the Band model the spectrum decreases as a power law. Item (i) implies directly that $E_{\mathrm{pk}}$ is likely overestimated when the CPL model is used in the spectral analysis, and items (ii) and (iii) imply that $E_{\gamma, \text { iso }}$, which is calculated in the rest-frame energy range of $1-10000 \mathrm{keV}$ by extrapolating the fitted spectrum, is likely underestimated.
In order to explore in a statistical sense the effect in the $E_{\mathrm{pk}}^{\prime}-E_{\gamma \text {,iso }}$ correlation of using the CPL model instead of the Band one for the spectral fitting, we carried out the following experiment. The time-averaged spectra of the 29 long GRBs that had a reasonable CPL-model fit (groups A and B) were fitted with XSPEC to a Band model, but with the high-energy power law index $\beta$ frozen to the typical value of -2.3 . As in the case of CPL, we have modified the Band model in order to perform the fit for the logarithms of the normalization and $E_{\mathrm{pk}}$. Then, we followed the same procedures described in $\S 2$ for handling the errors, taking into account the correlation among them. Fig. 5 shows the final data points and their $68 \%$ CL ellipses in the $E_{\mathrm{pk}}-E_{\gamma \text {,iso }}$ diagram. As expected, the normalization of the $E_{\mathrm{pk}}^{\prime}-E_{\gamma, \text { iso }}$ correlation decreases (with respect to Fig. 4), now being almost equal to the one of the updated Amati relation given in Ghirlanda (2007) for the range of $E_{\gamma \text {,iso values studied }}$ here. The best fit in logarithmic quantities is:

$$
\log \left(\frac{E_{\mathrm{pk}}^{\prime}}{\mathrm{keV}}\right)=(2.23 \pm 0.01)+(0.53 \pm 0.04) \log \left(\frac{E_{\gamma, \text { iso }}}{10^{52.61} \mathrm{erg}}\right)
$$

\section{SUMMARY AND CONCLUSIONS}

We have analysed homogeneously the time-integrated spectra of a relatively large sample of long-duration GRBs with known redshift obtained by a same detector, the Swift-BAT instrument. We have carried out spectral fits to 47 GRBs by using the two-parameter PL and three-parameter CPL photon models. Due to the narrow spectral range of BAT, 15-150 keV, only two- or three-parameter models can give reliable fits. However, even in these cases, large uncertainties are expected in the fitted parameters, making the study of the (highly correlated) errors important. We have performed Monte Carlo simulations in order to propagate the errors and calculate composite quantities such as $E_{\mathrm{pk}}^{\prime}$ and

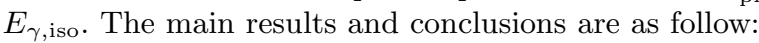

- For 29 GRBs (classes A+B) out of the 47 bursts analysed here, the CPL model, which identifies a peak energy $E_{\mathrm{pk}}$ in the $\nu f_{\nu}$ spectrum, gives reliable fits, though in 5 cases (class B), the best fit value for $E_{\mathrm{pk}}$ lies beyond the upper limit $(150 \mathrm{keV})$ of the BAT energy band. The uncertainties in the fitted parameters are in most cases highly correlated among them.

- The spectra of the remaining 18 bursts (classes $\mathrm{C}+\mathrm{D})$ are well fitted by the simple PL model. For 11 bursts (class C) of the $47 \mathrm{GRBs}$, the best PL-fit value of the photon index $\alpha$ is greater than -2 , suggesting that $E_{\mathrm{pk}}>150 \mathrm{keV}$. The other 7 bursts (class D) show a photon index $\alpha$ lower than -2 which implies a decreasing power-law $\nu f_{\nu}$ spectrum in the analysed energy range. In these cases, the peak energy could lie in the $\mathrm{X}$-ray range.

- The fluence, $S_{\gamma}$, and rest-frame isotropic equivalent energy, $E_{\gamma, \text { iso }}$, as well as their uncertainties, were calculated for the 29 (classes A+B) GRBs with reliable fits to the CPL model. This is currently the largest homogeneous sample of long GRBs with determined spectral parameters and $E_{\gamma \text {,iso }}$. The mean values of the rest peak energy $E_{\mathrm{pk}}^{\prime}$ and $E_{\gamma \text {,iso }}$ in the sample are $\sim 350 \mathrm{keV}$ and $\sim 4 \times 10^{52} \mathrm{erg}$, respectively.

- The $E_{\mathrm{pk}}$ as inferred from the CPL fit and $\alpha_{\mathrm{PL}}$ as inferred from the PL fit do correlate. However, this correlation 


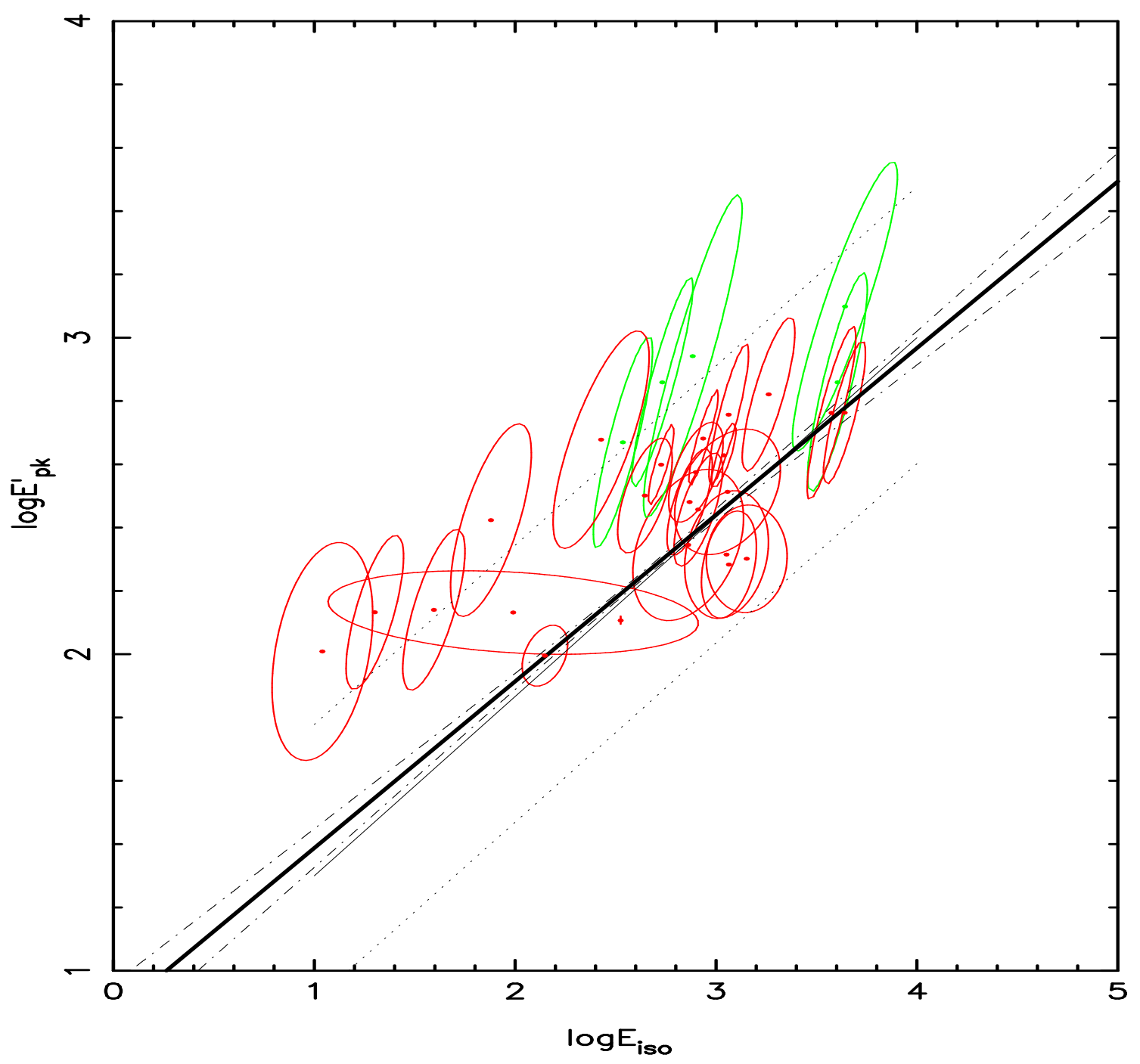

Figure 5. Same as Fig. 4 but for $E_{\mathrm{pk}}^{\prime}$ and $E_{\gamma \text {,iso }}$ inferred from spectral fits using the Band model with $\beta$ frozen to -2.3 . Note that using the Band model the correlation shifts to the higher (lower) $E_{\gamma, \text { iso }}\left(E_{\mathrm{pk}}^{\prime}\right)$ side with respect to the case when the CPL model is used.

is not physical, but is the result of the attempt of the single PL to account for the data points above the peak, with smaller flux. As $E_{\mathrm{pk}}$ decreases, a larger fraction of data lies above the peak, inducing the single power law to steepen. Therefore, the correlation should not be used to infer $E_{\mathrm{pk}}$ when knowing only $\alpha_{\mathrm{PL}}$. Indeed, we show that $E_{\mathrm{pk}}$ and $\alpha_{\mathrm{CPL}}$ do not correlate.

- A correlation between $E_{\mathrm{pk}}^{\prime}$ and $E_{\gamma \text {,iso }}$ (the 'Amati' relation) is confirmed for our sample [Eq. (5)]. This correlation and its scatter are consistent with the ones established previously for non-Swift bursts, showing that the $E_{\mathrm{pk}}^{\prime}-E_{\gamma \text {,iso }}$ correlation is hardly an artifact of selection effects. The zeropoint of our correlation (in $\log -\log$ ) is larger by $\sim 0.1$ dex at the sample median value of $E_{\gamma, \text { iso }}$ than the latest updated "Amati" correlation (Ghirlanda 2007). This difference is expected due to the use of a CPL model for describing the observed spectra instead of the Band model.

- When the Band model with the high-energy photon index frozen to $\beta=-2.3$ is fitted to the spectra of our 29 (classes $\mathrm{A}+\mathrm{B}) \mathrm{GRBs}$, the zero-point of the resultant $E_{\mathrm{pk}}^{\prime}$
$E_{\gamma, \text { iso }}$ correlation becomes smaller than in the case of the CPL model. For the Band model, the obtained $E_{\mathrm{pk}}^{\prime}-E_{\gamma \text {,iso }}$ correlation is given by Eq. (7), which is virtually indistinct from the 'Amati' correlation established previously for uneven observable data-sets from different satellites.

Although in this work we were able to process homogeneously the spectra of a significant fraction of the Swift GRBs with known redshift, the sample is still limited for conclusive results regarding the physical meaning of the correlations among the burst observable properties. During the refereeing process of this work, appeared a paper by Li (2007b), where the author finds evidence of significant change in the 'Amati' correlation parameters with redshift for the heterogeneous sample of 48 GRBs compiled by Amati (2006a). The number of GRBs with known redshifts and full spectral information obtained homogeneously should increase in order to attain more conclusive results. 


\section{ACKNOWLEDGMENTS}

JIC and VA-R gratefully acknowledge the hospitality extended by Osservatorio Astronomico di Brera in Merate during research stays. JIC is supported by a CONACyT (Mexico) fellowship. We are grateful to the anonymous Referee for his/her comments, which helped to correct some bugs in the processed data and to improve the manuscript. This work was supported by the PAPIIT-UNAM grant IN107706-3 to VA-R, and by an italian 2005 PRIN-INAF grant. The authors acknowledge the use of the Swift publicly available data as well as the public XSPEC software package. We thank J. Benda for grammar corrections to the manuscript.

\section{REFERENCES}

Amati, L., et al. 2002, A\&A, 390, 81

Amati, L. 2006a, MNRAS, 372, 233

Amati, L. 2006b, in proceedings of "SWIFT and GRBs: Unveiling the Relativistic Universe", preprint (astro-ph/0611189)

Atteia, J.-L. 2003, A\&A, 407, L1

Band, D., et al. 1993, ApJ, 413, 281

Barraud, C. et al. 2003, A\&A, 400, 1021

Barthelmy, S. D. et al. 2005, Spa. Sci. Rev. 120, 143

Bevington, P. R. \& Robinson, D. K. 1992, "Data reduction and error analysis for the Physical sciences", Mc-Graw Hill College

Crew G., Ricker G., Atteia J-L., et al. 2005, GCN 4021

Dai Z.G., Liang E.W. \& Xu D. 2004, ApJ, 612, L101

Firmani C., Avila-Reese V., Ghisellini G. \& Tutukov A.V. 2004, ApJ, 611, 1033

Firmani C., Ghisellini G., Ghirlanda G. \& Avila-Reese V., 2005, MNRAS, 360, L1

Firmani C., Ghisellini G., Avila-Reese V., Ghirlanda G., 2006a, MNRAS, 370, 185

Firmani C., Avila-Reese V., Ghisellini G., \& Ghirlanda G., 2006b, MNRAS, 372, L28

Firmani C., Avila-Reese V., Ghisellini G., \& Ghirlanda G., 2007, RevMexAA, 43, 203

Gehrels, N. et al. 2004, ApJ, 611, 1005

Ghirlanda, G., 2007, Phil.Trans.Roy.Soc.A.Proc., in press (astro-ph/0702212)

Ghirlanda, G., Ghisellini, G. \& Lazzati, D., 2004a, ApJ, 616,331

Ghirlanda G., Ghisellini G., Lazzati D. \& Firmani C., 2004b, ApJ, 613, L13

Ghirlanda G., Ghisellini G.,\& Firmani C., 2006, New J. Phys. 8, 123

Golenetskii S., Aptekar R., Mazets E., Pal'shin V., Frederiks D., \& Cline T. 2005a, GCN 3179

Golenetskii S., Aptekar R., Mazets E., Pal'shin V., Frederiks D., \& Cline T. 2005b, GCN 3518

Golenetskii S., Aptekar R., Mazets E., Pal'shin V., Frederiks D., \& Cline T. 2005c, GCN 4238

Guetta, D., Granot, J., \& Begelman, M. C. 2005, ApJ, 622, 482

Kaneko, Y., Preece, R. D., Briggs, M. S., Paciesas, W. S., Meegan, C. A., \& Band, D. L. 2006, ApJSS, 166, 298

Kippen, R. M., Woods, P. M., Heise, J., in't Zand, J., Preece, R. D., \& Briggs, M. S. 2001, Gamma-ray Bursts in the Afterglow Era, 22
Li, L.-X. 2007a, MNRAS, 374, L20

Li, L.-X. 2007b, MNRAS, 379, L55

Liang E. \& Zhang B., 2005, ApJ, 633, 611

Lloyd-Ronning, N. M., Fryer, C. L., \& Ramirez-Ruiz, E. 2002, ApJ, 574, 554

Mészáros, P. 2006, Reports of Progress in Physics, 69, 2259

Piran, T. 2005, Rev. Mod. Phys. 761143

Preece, R. D., Briggs, M. S., Mallozzi, R.S., Pendleton, G. N., Paciesas, W. S. \& Band, D. L., 2000, ApJSS, 126, 19

Press W.H. et al., 1999, Numerical Recipes in C, Cambridge University Press

Ramirez-Ruiz E. \& Granot J., 2006, preprint (astro-ph/0608379)

Sakamoto T. et al., 2005, ApJ, 629, 311

Schaefer, B. E. 2007, ApJ, 660, 16

Thompson, C. 2006, ApJ, 651, 333

Thompson, C., Rees, M. J. \& Mészáros, P. 2007, ApJ in press (astro-ph/0608282)

Yonetoku, D., Murakami, T., Nakamura, T., Yamazaki, R., Inoue, A. K., \& Ioka, K. 2004, ApJ, 609, 935

Xu D., Dai Z. G. \& Liang E. W., 2005, ApJ, 633, 603

Zhang, B. \& Mészáros, P. 2004, IJMPA, 19, 2385

Zhang, B. 2007, ChJAA, 7, 1

\section{APPENDIX A: THE OBSERVED SPECTRA}

In this Appendix section we present the 47 Swift spectra (see Table 1) analysed here. As described in $\S 2$, the 60 energy channels in the range $15-150 \mathrm{keV}$ of the BAT detector are used to deconvolve the spectra. The spectral fluxes were averaged over the integration time, $T_{\text {int }}$, determined for each burst from its light curve (see $\S 2$ ). In Figs. A1, A2 and A3. the CPL model was used to fit the observed spectra. 


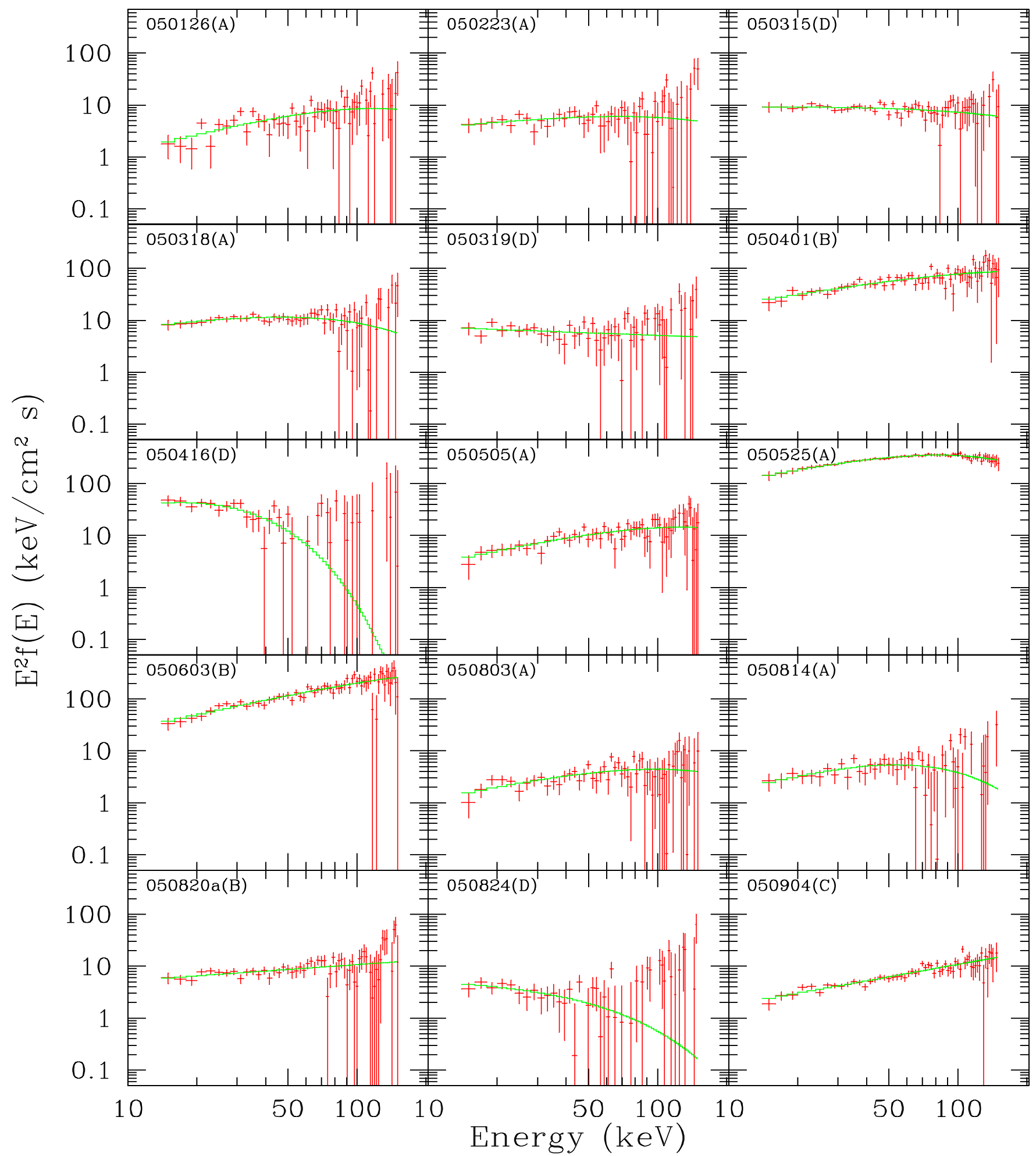

Figure A1. Time-averaged $\nu f_{\nu}$ spectra (red error bars) of the Swift GRBs with known redshift included in our sample. Continuous (green) line: best fit curve with the CPL photon model. The spectral flux of each burst was averaged over its integration time, $\mathrm{T}_{\mathrm{int}}$. 
Spectral analysis of Swift GRBs with known z

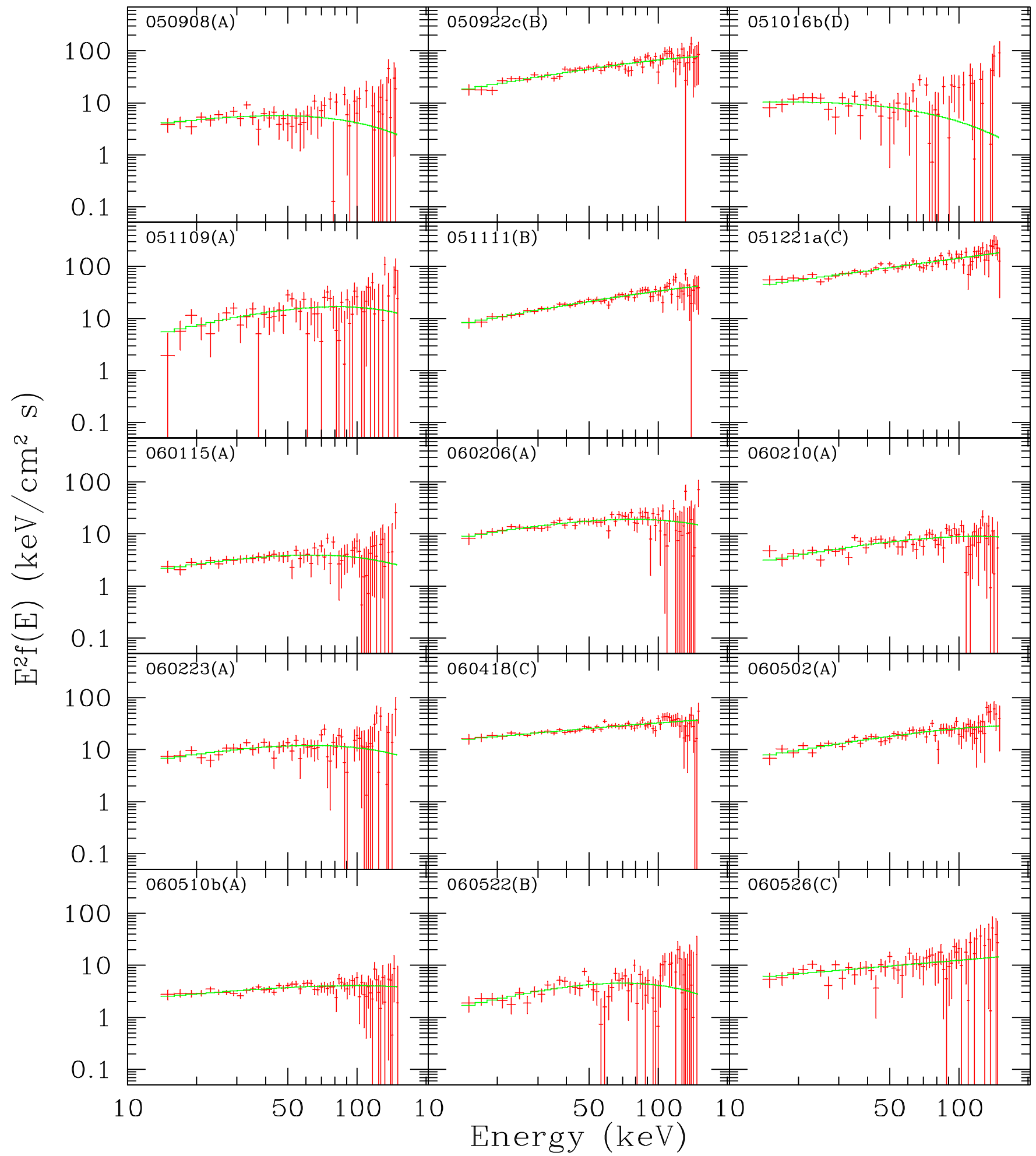

Figure A2. Continues Fig. A1 


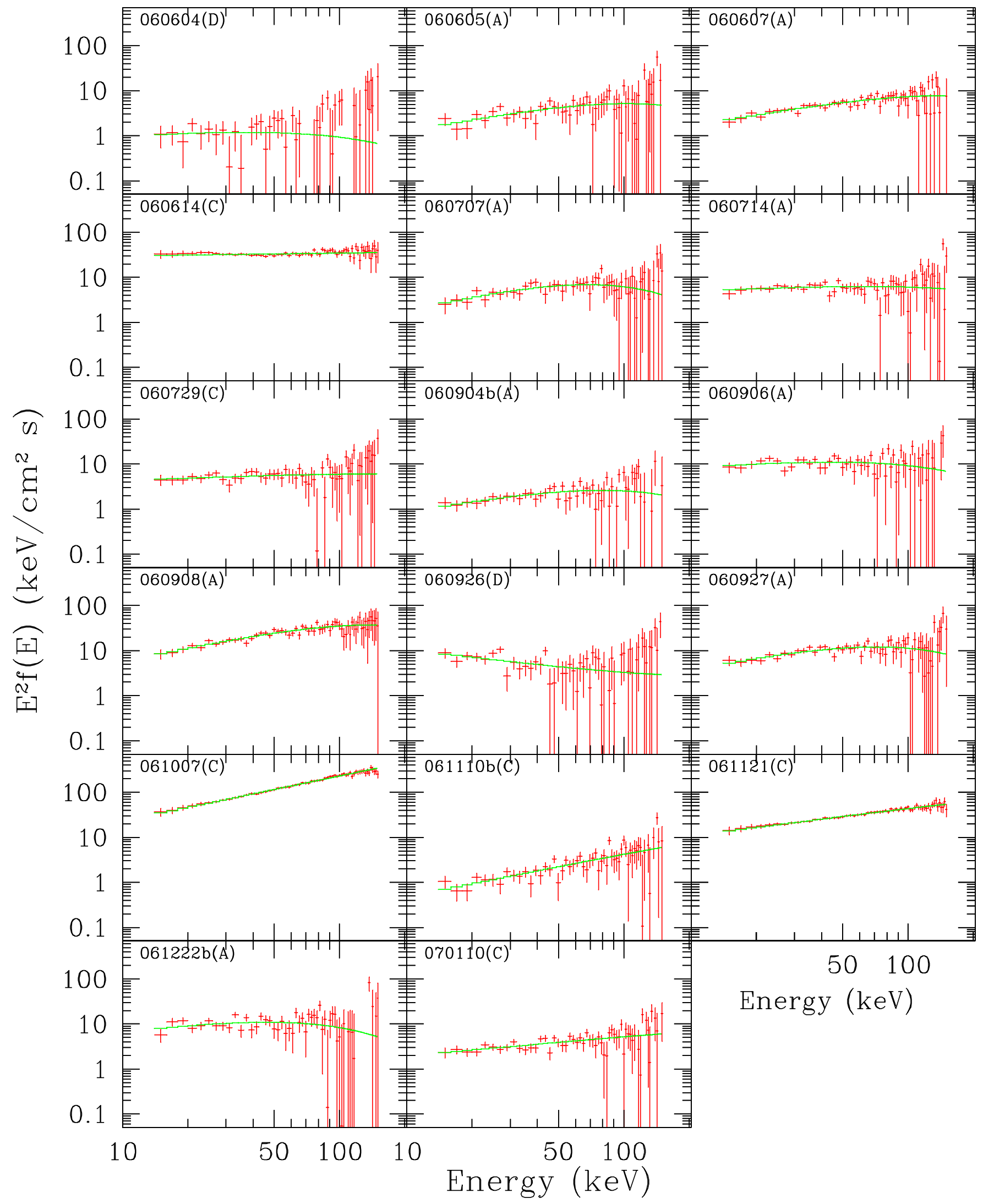

Figure A3. Continues Fig. A1 